\title{
Microcanonical conditioning of Markov processes on time-additive observables
}

\author{
Cécile Monthus \\ Université Paris Saclay, CNRS, CEA, Institut de Physique Théorique, 91191 Gif-sur-Yvette, France
}

\begin{abstract}
The recent study by B. De Bruyne, S. N. Majumdar, H. Orland and G. Schehr [J. Stat. Mech. (2021) 123204], concerning the conditioning of the Brownian motion and of random walks on global dynamical constraints over a finite time-window $T$, is reformulated as a general framework for the 'microcanonical conditioning' of Markov processes on time-additive observables. This formalism is applied to various types of Markov processes, namely discrete-time Markov chains, continuous-time Markov jump processes and diffusion processes in arbitrary dimension. In each setting, the timeadditive observable is also fully general, i.e. it can involve both the time spent in each configuration and the elementary increments of the Markov process. The various cases are illustrated via simple explicit examples. Finally, we describe the link with the 'canonical conditioning' based on the generating function of the time-additive observable for finite time $T$, while the regime of large time $T$ allows to recover the standard large deviation analysis of time-additive observables via the deformed Markov operator approach.
\end{abstract}

\section{INTRODUCTION}

Time-additive observables of Markov processes have attracted a lot of interest recently, in particular in the field of non equilibrium steady states in order to characterize their dynamical fluctuations over a large time-window $T$. From the point of view of the large deviation theory (see the reviews [1-3] and references therein), time-additive observables belong to the Level 1 and can be thus analyzed via the contraction from higher Levels. For instance, the large deviations at Level 2 for the empirical density allows to analyze the time-additive observables that only depend on the time spent in each configuration, but the Level 2 is usually not closed for non-equilibrium processes with steady currents. By contrast, the Level 2.5 concerning the joint distribution of the empirical density and of the empirical flows can be written in closed form for general Markov processes, including discrete-time Markov chains [3-8], continuoustime Markov jump processes [4, 7-28] and Diffusion processes [7, 8, 12, 13, 16, 26, 29-31]. In addition, this Level 2.5 is necessary to analyze via contraction the general case of time-additive observables that involve not only the time spent in each configuration but also the elementary increments of the Markov process. Another standard method to characterize the statistics of a time-additive observables is to study its generating function via the appropriate deformed Markov operator that does not conserve the probability [16, 31-74], while the probability-conserving Markov process corresponding to this 'canonical conditioning' can be written from the generalization of Doob's h-transform.

On the other hand, the 'microcanonical conditioning' of one-dimensional stochastic processes on time-additive observables has been considered recently in order to have efficient methods to generate stochastic trajectories satisfying global dynamical constraints over a finite time window $T$. The conditioning on the area has been studied via various methods for Brownian processes or bridges [75] and for Ornstein-Uhlenbeck bridges [76] (see also [77-79] for the discussion of other types of conditioning). The conditioning on the area and on other time-additive observables has been then analyzed for the Brownian motion and for discrete-time random walks [80], building on previous works [81-86] concerning the standard Doob conditioning, where the goal was to generate stochastic trajectories ending in a specific configuration at time $T$.

In the present paper, the approach of the recent study [80] is reformulated as a general framework for the 'microcanonical conditioning' of Markov processes on time-additive observables, where the Markov process can be either a discrete-time Markov chain, a continuous-time Markov jump process or a diffusion process in arbitrary dimension, while the time-additive observable can involve both the time spent in each configuration and the increments of the Markov process. This general formulation allows to make the link with the 'canonical conditioning' framework mentioned above.

The paper is organized as follows. In section II, we summarize the general ideas that allow to analyze the microcanonical conditioning of a Markov process on a time-additive observable. The specific applications to discrete-time Markov chains, to continuous-time Markov jump processes and to diffusion processes are then described in the sections III, IV and V respectively. Our conclusions are summarized in section VI. The links with the canonical conditioning on a time-additive observable are discussed for finite time $T$ in Appendix $\mathrm{A}$ and for large $T$ in the large deviation regime for the time-additive observable in Appendix B. 


\section{MICROCANONICAL CONDITIONING ON A TIME-ADDITIVE OBSERVABLE}

In this section, we summarize the general ideas and notations that will be useful in the whole paper. The equations will be written for discrete variables $(x, A)$, but the adaptation to continuous variables $(x, A)$ is of course straightforward : one just needs to replace sums by integrals, and discrete delta functions by continuous delta functions.

\section{A. Notion of time-additive observable $A(t)$ for the Markov process $x(t)$}

For the Markov process $x(t)$, the observable $A(t)$ is called time-additive if the difference $\left(A\left(t_{2}\right)-A\left(t_{1}\right)\right)$ between the value $A\left(t_{2}\right)$ at time $t_{2}$ and the value $A\left(t_{1}\right)$ at time $t_{1}$ is a deterministic function $\mathcal{A}[$.] of the Markov trajectory $x\left(t_{1} \leq s \leq t_{2}\right)$ between $s=t_{1}$ and $s=t_{2}$

$$
A\left(t_{2}\right)-A\left(t_{1}\right)=\mathcal{A}\left[x\left(t_{1} \leq s \leq t_{2}\right)\right]
$$

\section{B. Notion of microcanonical conditioning for the Markov process $x(t)$ and its time-additive observable $A(t)$}

In the main text, we will focus on the 'microcanonical conditioning' where both the initial values $\left(x_{0}, A_{0}\right)$ at time $t=0$ and the final values $\left(x_{T}, A_{T}\right)$ at time $t=T$ are fixed for the Markov process $x(t)$ and its time-additive observable $A(t)$. In order to analyze what happens at intermediate times $t \in[0, T]$, the approach described in [80] can be decomposed in the three steps described in the following three subsections.

\section{Joint propagator $P_{t, t_{0}}\left(x, A \mid x_{0}, A_{0}\right)$ for the Markov process and its time-additive observable}

The first step concerns the joint propagator $P_{t, t_{0}}\left(x, A \mid x_{0}, A_{0}\right)$ of the Markov process $x(t)$ and of its time-additive observable $A(t)$

$$
P_{t, t_{0}}\left(x, A \mid x_{0}, A_{0}\right) \equiv\left\langle\delta_{x(t), x} \delta_{A(t), A} \delta_{x\left(t_{0}\right), x_{0}} \delta_{A\left(t_{0}\right), A_{0}}\right\rangle
$$

Since the time-additive observable is a deterministic function $\mathcal{A}\left[\right.$.] of the Markov trajectory $x\left(t_{0} \leq s \leq t\right)($ see Eq. 1$)$, the joint propagator $P_{t, t_{0}}\left(x, A \mid x_{0}, A_{0}\right)$ satisfies :

(i) some Markov forward dynamics with respect to its final variables $(x, A)$ at time $t$

(ii) some Markov backward dynamics with respect to its initial variables $\left(x_{0}, A_{0}\right)$ at time $t_{0}$.

D. Conditional probability $\mathcal{P}_{t}^{C o n d}(x, A)$ if starting at $\left(x_{0}, A_{0}\right)$ at time $t=0$ and ending at $\left(x_{T}, A_{T}\right)$ at time $t=T$

The second step concerns the conditional probability $\mathcal{P}_{t}^{\text {Cond }}(x, A)$ to be at the values $(x, A)$ at some intermediate time $t \in] 0, T$ if starting at the values $\left(x_{0}, A_{0}\right)$ at time $t=0$ and ending at the values $\left(x_{T}, A_{T}\right)$ at time $t=T$. The probability $P_{T, 0}\left(x_{T}, A_{T} \mid x_{0}, A_{0}\right)$ to end at $\left(x_{T}, A_{T}\right)$ at time $t=T$ when starting at $\left(x_{0}, A_{0}\right)$ at time $t=0$ satisfies the Chapman-Kolmogorov equation with respect to any internal time $t \in] 0, T[$

$$
P_{T, 0}\left(x_{T}, A_{T} \mid x_{0}, A_{0}\right)=\sum_{x} \sum_{A} P_{T, t}\left(x_{T}, A_{T} \mid x, A\right) P_{t, 0}\left(x, A \mid x_{0}, A_{0}\right)
$$

So the conditional probability $\mathcal{P}_{t}^{C o n d}(x, A)$ to see the values $(x, A)$ at the internal time $\left.t \in\right] 0, T[$ is simply given by the ratio

$$
\mathcal{P}_{t}^{C o n d}(x, A)=\frac{P_{T, t}\left(x_{T}, A_{T} \mid x, A\right) P_{t, 0}\left(x, A \mid x_{0}, A_{0}\right)}{P_{T, 0}\left(x_{T}, A_{T} \mid x_{0}, A_{0}\right)}
$$

It is normalized as a consequence of Eq. 3

$$
\sum_{x} \sum_{A} \mathcal{P}_{t}^{\text {Cond }}(x, A)=1
$$


and it satisfies the fixed boundary conditions at time $t=0$ and at time $t=T$

$$
\begin{aligned}
& \mathcal{P}_{0}^{\text {Cond }}(x, A)=\frac{P_{T, 0}\left(x_{T}, A_{T} \mid x, A\right) P_{0,0}\left(x, A \mid x_{0}, A_{0}\right)}{P_{T, 0}\left(x_{T}, A_{T} \mid x_{0}, A_{0}\right)}=\delta_{x, x_{0}} \delta_{A, A_{0}} \\
& \mathcal{P}_{T}^{\text {Cond }}(x, A)=\frac{P_{T, T}\left(x_{T}, A_{T} \mid x, A\right) P_{T, 0}\left(x, A \mid x_{0}, A_{0}\right)}{P_{T, 0}\left(x_{T}, A_{T} \mid x_{0}, A_{0}\right)}=\delta_{x, x_{T}} \delta_{A, A_{T}}
\end{aligned}
$$

\section{E. Markov dynamics for the conditional probability $\mathcal{P}_{t}^{\text {Cond }}(x, A)$}

The third step consists in deriving the Markov dynamics of the conditional probability $\mathcal{P}_{t}^{C o n d}(x, A)$ from the Markov dynamics satisfied by the two joints propagators in the numerator of Eq. 4, namely :

(i) the Markov forward dynamics of the joint propagator $P_{t, 0}\left(x, A \mid x_{0}, A_{0}\right)$ with respect to its final variables $(x, A)$ at time $t$

(ii) the Markov backward dynamics of the joint propagator $P_{T, t}\left(x_{T}, A_{T} \mid x, A\right)$ with respect to its initial variables $(x, A)$ at time $t$

In the three following sections, the Markov dynamics for the conditional probability $\mathcal{P}_{t}^{C o n d}(x, A)$ is written explicitly for discrete-time Markov chains (section III), for continuous-time Markov jump processes (section IV) and for diffusion processes $(\mathrm{V})$.

\section{APPLICATION TO DISCRETE-TIME MARKOV CHAINS}

In this section, we focus on the Markov Chain dynamics where the probability $P_{t}(x)$ to be in the configuration $x$ at time $t$ evolves according to

$$
P_{t+1}(x)=\sum_{x^{\prime}} W\left(x ; x^{\prime}\right) P_{t}\left(x^{\prime}\right)
$$

The matrix element $W\left(x ; x^{\prime}\right) \in[0,1]$ represents the probability to be in the configuration $x$ at time $(t+1)$ if in the configuration $x^{\prime}$ at $t$, with the normalization for any $x^{\prime}$

$$
\sum_{x} W\left(x ; x^{\prime}\right)=1
$$

The time-additive observable $A(t)$ of the trajectory $x\left(t_{1} \leq s \leq t_{2}\right)$ of Eq. 1 can be parametrized by some function $\beta(x, y)$

$$
A\left(t_{2}\right)-A\left(t_{1}\right)=\mathcal{A}\left[x\left(t_{1} \leq s \leq t_{2}\right)\right] \equiv \sum_{s=t_{1}+1}^{t_{2}} \beta(x(s), x(s-1))
$$

Since the time $t$ and the space $x$ are both discrete, the equations will be written below for the case of a discrete variable $A$, but the adaptation to a continuous variable $A$ is of course straightforward : one just needs to replace sums by integrals, and discrete delta functions by continuous delta functions.

\section{A. Dynamics of the joint propagator $P_{t, t_{0}}\left(x, A \mid x_{0}, A_{0}\right)$}

Since the increment between $t$ and $(t+1)$ of the time-additive observable $A(t)$ of Eq. 9 reduces to

$$
A(t+1)-A(t)=\beta(x(t+1), x(t))
$$

one just needs to introduce the joint generator

$$
W\left(x, A ; x^{\prime}, A^{\prime}\right)=W\left(x ; x^{\prime}\right) \delta_{A, A^{\prime}+\beta\left(x, x^{\prime}\right)}
$$

that involves the initial Markov matrix $W\left(x ; x^{\prime}\right)$ of Eq. 7 , while the delta function in $A$ describes the deterministic evolution of the time-additive observable once the configurations $x$ and $x^{\prime}$ are given. The normalization of Eq. 8 ensures the normalization of the joint generator for any $\left(x^{\prime}, A^{\prime}\right)$

$$
\sum_{x} \sum_{A} W\left(x, A ; x^{\prime}, A^{\prime}\right)=\sum_{x} W\left(x ; x^{\prime}\right)=1
$$


The joint propagator $P_{t, t_{0}}\left(x, A \mid x_{0}, A_{0}\right)$ of Eq. 2 satisfies

(i) the forward dynamics with respect to the final variables $(x, A)$

$$
P_{t+1, t_{0}}\left(x, A \mid x_{0} A_{0}\right)=\sum_{x^{\prime}} \sum_{A^{\prime}} W\left(x, A ; x^{\prime}, A^{\prime}\right) P_{t, t_{0}}\left(x^{\prime}, A^{\prime} \mid x_{0} A_{0}\right)
$$

(ii) the backward dynamics with respect to the initial variables $\left(x_{0}, A_{0}\right)$

$$
P_{t, t_{0}-1}\left(x, A \mid x_{0} A_{0}\right)=\sum_{x_{0}^{\prime}} \sum_{A_{0}^{\prime}} P_{t, t_{0}}\left(x, A \mid x_{0}^{\prime} A_{0}^{\prime}\right) W\left(x_{0}^{\prime}, A_{0}^{\prime} ; x_{0}, A_{0}\right)
$$

\section{B. Forward Markov dynamics for the conditional probability $\mathcal{P}_{t}^{C o n d}(x, A)$ with a time-dependent generator}

Let us plug the forward dynamics of Eq. 13 for $P_{t+1}\left(x, A \mid x_{0}, A_{0}\right)$ into the conditional probability of Eq. 4 at time $(t+1)$

$$
\begin{aligned}
\mathcal{P}_{t+1}^{C o n d}(x, A) & =\frac{P_{T, t+1}\left(x_{T}, A_{T} \mid x, A\right)}{P_{T, 0}\left(x_{T}, A_{T} \mid x_{0}, A_{0}\right)} P_{t+1,0}\left(x, A \mid x_{0}, A_{0}\right) \\
& =\frac{P_{T, t+1}\left(x_{T}, A_{T} \mid x, A\right)}{P_{T, 0}\left(x_{T}, A_{T} \mid x_{0}, A_{0}\right)} \sum_{x^{\prime}} \sum_{A^{\prime}} W\left(x, A ; x^{\prime}, A^{\prime}\right) P_{t, 0}\left(x^{\prime}, A^{\prime} \mid x_{0} A_{0}\right)
\end{aligned}
$$

Let us now use the conditional probability at time $t$ of Eq. 4 to replace $P_{t, t_{0}}\left(x^{\prime}, A^{\prime} \mid x_{0} A_{0}\right)$

$$
P_{t, 0}\left(x^{\prime}, A^{\prime} \mid x_{0}, A_{0}\right)=\frac{P_{T, 0}\left(x_{T}, A_{T} \mid x_{0}, A_{0}\right)}{P_{T, t}\left(x_{T}, A_{T} \mid x^{\prime}, A^{\prime}\right)} \mathcal{P}_{t}^{C o n d}\left(x^{\prime}, A^{\prime}\right)
$$

in order to rewrite Eq. 15 as the forward Markov dynamics

$$
\begin{aligned}
\mathcal{P}_{t+1}^{C o n d}(x, A) & =\frac{P_{T, t+1}\left(x_{T}, A_{T} \mid x, A\right)}{P_{T, 0}\left(x_{T}, A_{T} \mid x_{0}, A_{0}\right)} \sum_{x^{\prime}} \int d A^{\prime} W\left(x, A ; x^{\prime}, A^{\prime}\right) \frac{P_{T, 0}\left(x_{T}, A_{T} \mid x_{0}, A_{0}\right)}{P_{T, t}\left(x_{T}, A_{T} \mid x^{\prime}, A^{\prime}\right)} \mathcal{P}_{t}^{C o n d}\left(x^{\prime}, A^{\prime}\right) \\
& \equiv \sum_{x^{\prime}} \int d A^{\prime} W_{t+1 / 2}^{\text {Forw }\left[x_{T}, A_{T} ; T\right]}\left(x, A ; x^{\prime}, A^{\prime}\right) \mathcal{P}_{t}^{C o n d}\left(x^{\prime}, A^{\prime}\right)
\end{aligned}
$$

where the generator associated to this forward conditioned dynamics

$$
W_{t+1 / 2}^{F o r w\left[x_{T}, A_{T} ; T\right]}\left(x, A ; x^{\prime}, A^{\prime}\right) \equiv P_{T, t+1}\left(x_{T}, A_{T} \mid x, A\right) W\left(x, A ; x^{\prime}, A^{\prime}\right) \frac{1}{P_{T, t}\left(x_{T}, A_{T} \mid x^{\prime}, A^{\prime}\right)}
$$

is time-dependent because the joint generator $W\left(x, A ; x^{\prime}, A^{\prime}\right)$ of Eq. 11 is conjugated with the full propagators $P_{T, t+1}\left(x_{T}, A_{T} \mid x, A\right)$ and $P_{T, t}\left(x_{T}, A_{T} \mid x^{\prime}, A^{\prime}\right)$ up to the imposed final values $\left(x_{T}, A_{T}\right)$ at time $T$. The normalization for any $\left(x^{\prime}, A^{\prime}\right)$ of this conditional forward generator

$$
\sum_{x} \sum_{A} W_{t+1 / 2}^{\text {Forw }\left[x_{T}, A_{T} ; T\right]}\left(x, A ; x^{\prime}, A^{\prime}\right)=1
$$

is ensured by the backward recursion of Eq. 14 .

The physical meaning of the generator of Eq. 18 is that in the conditioned dynamics, the possibles transitions are the same as in the initial dynamics (an impossible transition $W\left(x, A ; x^{\prime}, A^{\prime}\right)=0$ in the initial dynamics remains impossible $W_{t+1 / 2}^{\text {Forw }\left[x_{T}, A_{T} ; T\right]}\left(x, A ; x^{\prime}, A^{\prime}\right)=0$ in the conditioned dynamics), but the possible transitions have different probabilities that have changed from $W\left(x, A ; x^{\prime}, A^{\prime}\right)$ to $W_{t+1 / 2}^{F o r w\left[x_{T}, A_{T} ; T\right]}\left(x, A ; x^{\prime}, A^{\prime}\right)$.

In practice, if one wishes to use these new probabilities $\left.W_{t+1 / 2}^{F o r w ~} x_{T}, A_{T} ; T\right]\left(x, A ; x^{\prime}, A^{\prime}\right)$ to generate stochastic trajectories of the conditioned dynamics, one needs to know the explicit form of the joint propagator $P_{t, t_{0}}\left(x, A \mid x_{0}, A_{0}\right)$ of Eq. 2 satisfying the joint forward dynamics of Eq. 13. 
C. Backward Markov dynamics for the conditional probability $\mathcal{P}_{t}^{C o n d}(x, A)$ with a time-dependent generator

Let us write the backward recursion of Eq. 14 for $P_{T, t}\left(x_{T}, A_{T} \mid x, A\right)$

$$
P_{T, t}\left(x_{T}, A_{T} \mid x, A\right)=\sum_{x^{\prime}} \sum_{A^{\prime}} P_{T, t+1}\left(x_{T}, A_{T} \mid x^{\prime}, A^{\prime}\right) W\left(x^{\prime}, A^{\prime} ; x, A\right)
$$

and use the conditional probability of Eq. 4 at time $(t+1)$ to make the replacement

$$
P_{T, t+1}\left(x_{T}, A_{T} \mid x^{\prime}, A^{\prime}\right)=\mathcal{P}_{t+1}^{C o n d}\left(x^{\prime}, A^{\prime}\right) \frac{P_{T, 0}\left(x_{T}, A_{T} \mid x_{0}, A_{0}\right)}{P_{t+1,0}\left(x^{\prime}, A^{\prime} \mid x_{0}, A_{0}\right)}
$$

into order to rewrite the conditional probability of Eq. 4 as

$$
\begin{aligned}
\mathcal{P}_{t}^{\text {Cond }}(x, A) & =\sum_{x^{\prime}} \sum_{A^{\prime}} P_{T, t+1}\left(x_{T}, A_{T} \mid x^{\prime}, A^{\prime}\right) \frac{P_{t, 0}\left(x, A \mid x_{0}, A_{0}\right)}{P_{T, 0}\left(x_{T}, A_{T} \mid x_{0}, A_{0}\right)} \\
& =\sum_{x^{\prime}} \sum_{A^{\prime}} \mathcal{P}_{t+1}^{\text {Cond }}\left(x^{\prime}, A^{\prime}\right) \frac{P_{T, 0}\left(x_{T}, A_{T} \mid x_{0}, A_{0}\right)}{P_{t+1,0}\left(x^{\prime}, A^{\prime} \mid x_{0}, A_{0}\right)} W\left(x^{\prime}, A^{\prime} ; x, A\right) \frac{P_{t, 0}\left(x, A \mid x_{0}, A_{0}\right)}{P_{T, 0}\left(x_{T}, A_{T} \mid x_{0}, A_{0}\right)} \\
& \equiv \sum_{x^{\prime}} \sum_{A^{\prime}} \mathcal{P}_{t+1}^{\text {Cond }}\left(x^{\prime}, A^{\prime}\right) W_{t+1 / 2}^{B a c k w\left[x_{0}, A_{0} ; 0\right]}\left(x^{\prime}, A^{\prime} ; x, A\right)
\end{aligned}
$$

where the generator associated to this backward conditioned dynamics

$$
W_{t+1 / 2}^{B a c k w\left[x_{0}, A_{0} ; 0\right]}\left(x^{\prime}, A^{\prime} ; x, A\right) \equiv \frac{1}{P_{t+1,0}\left(x^{\prime}, A^{\prime} \mid x_{0}, A_{0}\right)} W\left(x^{\prime}, A^{\prime} ; x, A\right) P_{t, 0}\left(x, A \mid x_{0}, A_{0}\right)
$$

involves the conjugation of joint generator $W\left(x^{\prime}, A^{\prime} ; x, A\right)$ of Eq. 11 by the full propagators $P_{t+1,0}\left(x^{\prime}, A^{\prime} \mid x_{0}, A_{0}\right)$ and $P_{t, 0}\left(x, A \mid x_{0}, A_{0}\right)$ up to the imposed initial values $\left(x_{0}, A_{0}\right)$ at time $t=0$.

\section{Illustration with a simple example : conditioning the Sisyphus Random Walk on the number of resets}

In the field of stochastic resetting (see the review [87] and references therein), one of the simplest example is the Sisyphus Random Walk [88] defined on the semi-infinite lattice $x=0,1,2, .$. with the Markov matrix

$$
W\left(x ; x^{\prime}\right)=R \delta_{x, 0}+(1-R) \delta_{x, x^{\prime}+1}
$$

The physical meaning is that when Sisyphus is at position $x$ at time $t$, he can either return to the origin $x=0$ with the reset probability $R \in$ ] 0,1 [ or he can move forward to the next position $(x+1)$ with the complementary probability $(1-R)$.

As time-additive observable of Eq. 9, we will choose the function

$$
\beta\left(x, x^{\prime}\right)=\delta_{x, 0}
$$

in order to count the total number of resets to the origin during the time interval

$$
A\left(t_{2}\right)-A\left(t_{1}\right)=\mathcal{A}\left[x\left(t_{1} \leq s \leq t_{2}\right)\right] \equiv \sum_{s=t_{1}+1}^{t_{2}} \delta_{x(s), 0}
$$

1. Explicit form of the joint propagator $P_{t, t_{0}}\left(x, A \mid x_{0} A_{0}\right)$

The joint generator of Eq. 11

$$
W\left(x, A ; x^{\prime}, A^{\prime}\right)=W\left(x ; x^{\prime}\right) \delta_{A, A^{\prime}+\delta_{x, 0}}=R \delta_{x, 0} \delta_{A, A^{\prime}+1}+(1-R) \delta_{x, x^{\prime}+1} \delta_{A, A^{\prime}}
$$


governs the forward dynamics of Eq. 13 for the joint propagator

$$
\begin{aligned}
P_{t+1, t_{0}}\left(x, A \mid x_{0} A_{0}\right) & =\sum_{x^{\prime}=0}^{+\infty} \sum_{A^{\prime}=A_{0}}^{A} W\left(x, A ; x^{\prime}, A^{\prime}\right) P_{t, t_{0}}\left(x^{\prime}, A^{\prime} \mid x_{0} A_{0}\right) \\
& =R \delta_{x, 0} \theta\left(A>A_{0}\right) \sum_{x^{\prime}=0}^{+\infty} P_{t, t_{0}}\left(x^{\prime}, A-1 \mid x_{0} A_{0}\right)+\theta(x>0)(1-R) P_{t, t_{0}}\left(x-1, A \mid x_{0} A_{0}\right)
\end{aligned}
$$

where the notation $\theta$ is used to denote the inequalities that need to be satisfied. The solution can be directly written from the renewal analysis of the dynamics

$$
\begin{aligned}
& P_{t, t_{0}}\left(x, A \mid x_{0} A_{0}\right)=(1-R)^{\left(t-t_{0}\right)} \delta_{A, A_{0}} \delta_{x, x_{0}+\left(t-t_{0}\right)} \\
& +R^{A-A_{0}}(1-R)^{\left(t-t_{0}\right)-\left(A-A_{0}\right)} \frac{\left[\left(t-t_{0}\right)-x-1\right] !}{\left[\left(A-A_{0}\right)-1\right] !\left[\left(t-t_{0}\right)-\left(A-A_{0}\right)-x\right] !} \theta\left(1 \leq A-A_{0} \leq\left(t-t_{0}\right)-x\right)
\end{aligned}
$$

The summation of Eq. 29 over the variable $x$ allows to recover that the propagator for the variable $A$ alone corresponds to the binomial distribution for $\left(A-A_{0}\right)$ and is independent of $x_{0}$

$$
\begin{aligned}
\sum_{x=0}^{+\infty} P_{t, t_{0}}\left(x, A \mid x_{0} A_{0}\right) & =R^{A-A_{0}}(1-R)^{\left(t-t_{0}\right)-\left(A-A_{0}\right)} \frac{\left(t-t_{0}\right) !}{\left(A-A_{0}\right) !\left[\left(t-t_{0}\right)-\left(A-A_{0}\right)\right] !} \theta\left(0 \leq A-A_{0} \leq\left(t-t_{0}\right)\right) \\
& \equiv P_{t, t_{0}}\left(A \mid A_{0}\right)
\end{aligned}
$$

The summation of Eq. 29 over the variable $A$ yields the propagator for the initial Sisyphus random walk $x(t)$ alone

$$
\sum_{A=A_{0}}^{+\infty} P_{t, t_{0}}\left(x, A \mid x_{0} A_{0}\right) \quad=(1-R)^{\left(t-t_{0}\right)} \delta_{x, x_{0}+\left(t-t_{0}\right)}+R(1-R)^{x} \theta\left(0 \leq x \leq\left(t-t_{0}\right)-1\right) \equiv P_{t, t_{0}}\left(x \mid x_{0}\right)
$$

that converges towards the steady state corresponding to the geometric distribution

$$
P_{t, t_{0}}\left(x \mid x_{0}\right) \underset{\left(t-t_{0}\right) \rightarrow+\infty}{\simeq} R(1-R)^{x} \theta(0 \leq x) \equiv P_{s t}(x)
$$

\section{Forward generator of the conditioned dynamics : resetting probabilities depending on time and configuration}

For the conditioned dynamics, the forward generator of Eq. 18 reads using the joint generator of Eq. 24

$$
\begin{aligned}
W_{t+1 / 2}^{F o r w\left[x_{T}, A_{T} ; T\right]}\left(x, A ; x^{\prime}, A^{\prime}\right) & =P_{T, t+1}\left(x_{T}, A_{T} \mid x, A\right) W\left(x, A ; x^{\prime}, A^{\prime}\right) \frac{1}{P_{T, t}\left(x_{T}, A_{T} \mid x^{\prime}, A^{\prime}\right)} \\
& =R \delta_{x, 0} \delta_{A, A^{\prime}+1} \frac{P_{T, t+1}\left(x_{T}, A_{T} \mid 0, A\right)}{P_{T, t}\left(x_{T}, A_{T} \mid x^{\prime}, A^{\prime}\right)}+(1-R) \delta_{x, x^{\prime}+1} \delta_{A, A^{\prime}} \frac{P_{T, t+1}\left(x_{T}, A_{T} \mid x, A\right)}{P_{T, t}\left(x_{T}, A_{T} \mid x^{\prime}, A\right)} \\
& \equiv \delta_{x, 0} \delta_{A, A^{\prime}+1} R_{t+1 / 2}^{F o r w\left[x_{T}, A_{T} ; T\right]}\left(x^{\prime}, A^{\prime}\right)+\delta_{x, x^{\prime}+1} \delta_{A, A^{\prime}}\left(1-R_{t+1 / 2}^{F o r w\left[x_{T}, A_{T} ; T\right]}\left(x^{\prime}, A^{\prime}\right)\right)
\end{aligned}
$$

where the effective resetting probability towards the origin $x=0$ depends on the time $t$ and on the values $\left(x^{\prime}, A^{\prime}\right)$ at time $t$ via

$$
R_{t+1 / 2}^{F o r w\left[x_{T}, A_{T} ; T\right]}\left(x^{\prime}, A^{\prime}\right)=R \frac{P_{T, t+1}\left(x_{T}, A_{T} \mid 0, A^{\prime}+1\right)}{P_{T, t}\left(x_{T}, A_{T} \mid x^{\prime}, A^{\prime}\right)}
$$

One can plug the explicit form of Eq. 29 for the joint propagator to obtain the explicit form of the effective resetting probability of Eq. 33 .

In summary, the conditioned dynamics corresponds to a Sisyphus random walk with modified resetting probabilities: when Sisyphus is in the configuration $\left(x^{\prime}, A^{\prime}\right)$ at time $t$, he can either return to the origin $x=0$ and increment the observable $A=A^{\prime}+1$ with the reset probability $R_{t+1 / 2}^{F o r w\left[x_{T}, A_{T} ; T\right]}\left(x^{\prime}, A^{\prime}\right)$ or he can move forward to the next position $\left(x^{\prime}+1\right)$ and keep the observable $A=A^{\prime}$ with the complementary probability $\left[1-R_{t+1 / 2}^{F o r w\left[x_{T}, A_{T} ; T\right]}\left(x^{\prime}, A^{\prime}\right)\right]$.

The 'canonical conditioning' (see the reminder in the two Appendices) of the Sisyphus Random Walk has been studied in [7] for the more general case where the reset probabilities of the initial model are space-dependent $R_{x}$ (instead of being given by the constant value $R$ ) and where the time-additive observable involves an arbitrary function $\beta\left(x, x^{\prime}\right)$.

As a final remark, let us stress that other explicit examples of microcanonical conditioning for discrete-time random walks on time-additive observables can be found in [80]. 


\section{APPLICATION TO CONTINUOUS-TIME MARKOV JUMP PROCESSES}

In this section, we consider the continuous-time dynamics in discrete configuration space defined by the Master Equation

$$
\partial_{t} P_{t}(x)=\sum_{x^{\prime} \neq x}\left[w\left(x ; x^{\prime}\right) P_{t}\left(x^{\prime}\right)-w\left(x^{\prime} ; x\right) P_{t}(x)\right]
$$

where $w\left(x ; x^{\prime}\right) \geq 0$ represents the transition rate from $x^{\prime}$ towards $x \neq x^{\prime}$.

The time-additive observable $A(t)$ of the trajectory $x\left(t_{1} \leq s \leq t_{2}\right)$ of Eq. 1 can be parametrized by the two functions $\alpha(x)$ and $\beta(x, y)$

$$
A\left(t_{2}\right)-A\left(t_{1}\right)=\mathcal{A}\left[x\left(t_{1} \leq s \leq t_{2}\right)\right]=\int_{t_{1}}^{t_{2}} d s \alpha(x(s))+\sum_{s \in\left[t_{1}, t_{2}\right]: x\left(s^{+}\right) \neq x(s)} \beta\left(x\left(s^{+}\right), x(s)\right)
$$

Whenever the function $\alpha($.$) is present, the observable A$ is continuous, so the equations will be written for continuous $A$ in the following general subsections, while an example with discrete variable $A$ will be given in the last subsection.

\section{A. Dynamics of the joint propagator $P_{t, t_{0}}\left(x, A \mid x_{0}, A_{0}\right)$}

Between $t$ and $(t+d t)$, the elementary increment of the time-additive observable of Eq. 36 reduces to

$$
A(t+d t)-A(t)=d t \alpha(x(t))+\delta_{x(t+d t) \neq x(t)} \beta(x(t+d t), x(t))
$$

As a consequence, the function $\alpha($.$) corresponds to a deterministic drift for the continuous observable A$, while the function $\beta(.,$.$) will appear via the following delta function in the joint jump rates from x^{\prime}$ to $x \neq x^{\prime}$

$$
w\left(x, A ; x^{\prime}, A^{\prime}\right) \equiv w\left(x ; x^{\prime}\right) \delta\left(A-A^{\prime}-\beta\left(x, x^{\prime}\right)\right)
$$

So the joint propagator $P_{t, t_{0}}\left(x, A \mid x_{0}, A_{0}\right)$ of Eq. 2 satisfies :

(i) the forward jump-drift dynamics with respect to the final values $(x, A)$ at time $t$

$$
\begin{aligned}
\partial_{t} P_{t, t_{0}}\left(x, A \mid x_{0}, A_{0}\right) & =-\partial_{A}\left[\alpha(x) P_{t, t_{0}}\left(x, A \mid x_{0}, A_{0}\right)\right] \\
& +\sum_{x^{\prime} \neq x} \int d A^{\prime}\left[w\left(x, A ; x^{\prime}, A^{\prime}\right) P_{t, t_{0}}\left(x^{\prime}, A^{\prime} \mid x_{0}, A_{0}\right)-w\left(x^{\prime}, A^{\prime} ; x, A\right) P_{t, t_{0}}\left(x, A \mid x_{0}, A_{0}\right)\right]
\end{aligned}
$$

(ii) the backward jump-drift dynamics with respect to the initial values $\left(x_{0}, A_{0}\right)$ at time $t_{0}$

$$
\begin{aligned}
-\partial_{t_{0}} P_{t, t_{0}}\left(x, A \mid x_{0}, A_{0}\right) & =\alpha\left(x_{0}\right) \partial_{A_{0}} P_{t, t_{0}}\left(x, A \mid x_{0}, A_{0}\right) \\
& +\sum_{x_{0}^{\prime} \neq x_{0}} \int d A_{0}^{\prime}\left[P_{t, t_{0}}\left(x, A \mid x_{0}^{\prime}, A_{0}^{\prime}\right)-P_{t, t_{0}}\left(x, A \mid x_{0}, A_{0}\right)\right] w\left(x_{0}^{\prime}, A_{0}^{\prime} ; x_{0}, A_{0}\right)
\end{aligned}
$$

\section{B. Markov dynamics for the conditional probability $\mathcal{P}_{t}^{C o n d}(x, A)$ with a time-dependent generator}

Let us now focus on the dynamics for the conditional probability $\mathcal{P}_{t}^{\text {Cond }}(x, A)$ of Eq. 4 . Its time-derivative involves the derivatives of the two propagators of the numerator

$$
\partial_{t} \mathcal{P}_{t}^{\text {Cond }}(x, A)=\left[\partial_{t} P_{T, t}\left(x_{T}, A_{T} \mid x, A\right)\right] \frac{P_{t, 0}\left(x, A \mid x_{0}, A_{0}\right)}{P_{T, 0}\left(x_{T}, A_{T} \mid x_{0}, A_{0}\right)}+\frac{P_{T, t}\left(x_{T}, A_{T} \mid x, A\right)}{P_{T, 0}\left(x_{T}, A_{T} \mid x_{0}, A_{0}\right)}\left[\partial_{t} P_{t, 0}\left(x, A \mid x_{0}, A_{0}\right)\right]
$$

Since the propagator $P_{t, 0}\left(x, A \mid x_{0}, A_{0}\right)$ satisfies the forward dynamics of Eq. 39, and since the propagator $P_{T, t}\left(x_{T}, A_{T} \mid x, A\right)$ satisfies the backward dynamics of Eq. 40

$$
\begin{aligned}
-\partial_{t} P_{T, t}\left(x_{T}, A_{T} \mid x, A\right) & =\alpha(x) \partial_{A} P_{T, t}\left(x_{T}, A_{T} \mid x, A\right) \\
& +\sum_{x^{\prime} \neq x} \int d A^{\prime}\left[P_{T, t}\left(x_{T}, A_{T} \mid x^{\prime}, A^{\prime}\right)-P_{T, t}\left(x_{T}, A_{T} \mid x, A\right)\right] w\left(x^{\prime}, A^{\prime} ; x, A\right)
\end{aligned}
$$


Eq. 41 becomes

$$
\begin{aligned}
\partial_{t} \mathcal{P}_{t}^{\text {Cond }}(x, A) & =-\alpha(x) \frac{P_{t, 0}\left(x, A \mid x_{0}, A_{0}\right)}{P_{T, 0}\left(x_{T}, A_{T} \mid x_{0}, A_{0}\right)} \partial_{A} P_{T, t}\left(x_{T}, A_{T} \mid x, A\right)-\alpha(x) \frac{P_{T, t}\left(x_{T}, A_{T} \mid x, A\right)}{P_{T, 0}\left(x_{T}, A_{T} \mid x_{0}, A_{0}\right)} \partial_{A} P_{t, 0}\left(x, A \mid x_{0}, A_{0}\right) \\
& -\frac{P_{t, 0}\left(x, A \mid x_{0}, A_{0}\right)}{P_{T, 0}\left(x_{T}, A_{T} \mid x_{0}, A_{0}\right)} \sum_{x^{\prime} \neq x} \int d A^{\prime} P_{T, t}\left(x_{T}, A_{T} \mid x^{\prime}, A^{\prime}\right) w\left(x^{\prime}, A^{\prime} ; x, A\right) \\
& +\frac{P_{T, t}\left(x_{T}, A_{T} \mid x, A\right)}{P_{T, 0}\left(x_{T}, A_{T} \mid x_{0}, A_{0}\right)} \sum_{x^{\prime} \neq x} \int d A^{\prime} w\left(x, A ; x^{\prime}, A^{\prime}\right) P_{t, 0}\left(x^{\prime}, A^{\prime} \mid x_{0}, A_{0}\right)
\end{aligned}
$$

(i) Forward perspective : Eq. 4 allows to replace all the propagators on $[0, t]$

$$
P_{t, 0}\left(x, A \mid x_{0}, A_{0}\right)=\mathcal{P}_{t}^{C o n d}(x, A) \frac{P_{T, 0}\left(x_{T}, A_{T} \mid x_{0}, A_{0}\right)}{P_{T, t}\left(x_{T}, A_{T} \mid x, A\right)}
$$

in Eq. 43 to obtain the forward dynamics

$$
\begin{aligned}
& \partial_{t} \mathcal{P}_{t}^{C o n d}(x, A)=-\alpha(x) \partial_{A} \mathcal{P}_{t}^{C o n d}(x, A) \\
& +\sum_{x^{\prime} \neq x} \int d A^{\prime}\left[w_{t}^{\text {Forw }\left[x_{T}, A_{T} ; T\right]}\left(x, A ; x^{\prime}, A^{\prime}\right) \mathcal{P}_{t}^{C o n d}\left(x^{\prime}, A^{\prime}\right)-w_{t}^{F o r w\left[x_{T}, A_{T} ; T\right]}\left(x^{\prime}, A^{\prime} ; x, A\right) \mathcal{P}_{t}^{C o n d}(x, A)\right]
\end{aligned}
$$

The difference with respect to the initial forward joint dynamics of Eq. 39 is in the time-dependent forward rates

$$
w_{t}^{F o r w\left[x_{T}, A_{T} ; T\right]}\left(x, A ; x^{\prime}, A^{\prime}\right) \equiv P_{T, t}\left(x_{T}, A_{T} \mid x, A\right) w\left(x, A ; x^{\prime}, A^{\prime}\right) \frac{1}{P_{T, t}\left(x_{T}, A_{T} \mid x^{\prime}, A^{\prime}\right)}
$$

that involve the conjugation of the joint rates $w\left(x, A ; x^{\prime}, A^{\prime}\right) \equiv w\left(x ; x^{\prime}\right) \delta\left(A-A^{\prime}-\beta\left(x, x^{\prime}\right)\right)$ of Eq. 38 with the full propagators $P_{T-t-1}\left(x_{T}, A_{T} \mid x, A\right)$ and $P_{T, t}\left(x_{T}, A_{T} \mid x^{\prime}, A^{\prime}\right)$ up to the imposed final values $\left(x_{T}, A_{T}\right)$ at time $T$. Eq. 46 is the analog of Eq. 18 concerning discrete-time Markov chains.

(ii) Backward perspective : Eq. 4 allows to replace all the propagators on $[t, T]$

$$
P_{T, t}\left(x_{T}, A_{T} \mid x, A\right)=\mathcal{P}_{t}^{C o n d}(x, A) \frac{P_{T, 0}\left(x_{T}, A_{T} \mid x_{0}, A_{0}\right)}{P_{t, 0}\left(x, A \mid x_{0}, A_{0}\right)}
$$

in Eq. 43 to obtain

$$
\begin{aligned}
& -\partial_{t} \mathcal{P}_{t}^{\text {Cond }}(x, A)=\alpha(x) \partial_{A} \mathcal{P}_{t}^{\text {Cond }}(x, A) \\
& +\sum_{x^{\prime} \neq x} \int d A^{\prime}\left[\mathcal{P}_{t}^{\text {Cond }}\left(x^{\prime}, A^{\prime}\right) w_{t}^{\text {Backw }\left[x_{0}, A_{0} ; 0\right]}\left(x^{\prime}, A^{\prime} ; x, A\right)-\mathcal{P}_{t}^{\text {Cond }}(x, A) w_{t}^{\text {Backw }\left[x_{0}, A_{0} ; 0\right]}\left(x, A ; x^{\prime}, A^{\prime}\right)\right]
\end{aligned}
$$

where the time-dependent backward rates

$$
w_{t}^{B a c k w\left[x_{0}, A_{0} ; 0\right]}\left(x^{\prime}, A^{\prime} ; x, A\right) \equiv \frac{1}{P_{t, 0}\left(x^{\prime}, A^{\prime} \mid x_{0}, A_{0}\right)} w\left(x^{\prime}, A^{\prime} ; x, A\right) P_{t, 0}\left(x, A \mid x_{0}, A_{0}\right)
$$

involve the conjugation of the joint rates $w\left(x, A ; x^{\prime}, A^{\prime}\right) \equiv w\left(x ; x^{\prime}\right) \delta\left(A-A^{\prime}-\beta\left(x, x^{\prime}\right)\right)$ of Eq. 38 with the full propagators $P_{t, 0}\left(x^{\prime}, A^{\prime} \mid x_{0}, A_{0}\right)$ and $P_{t, 0}\left(x, A \mid x_{0}, A_{0}\right)$ up to the imposed initial values $\left(x_{0}, A_{0}\right)$ at time $t=0$. Eq. 49 is the analog of Eq. 23 concerning discrete-time Markov chains.

(iii) The compatibility between the two dynamical equations of Eqs 45 and 48 can be checked via their sum

$$
\begin{aligned}
& 0 \stackrel{?}{=} \sum_{x^{\prime} \neq x} \int d A^{\prime}\left[w_{t}^{\text {Forw }\left[x_{T}, A_{T} ; T\right]}\left(x, A ; x^{\prime}, A^{\prime}\right) \mathcal{P}_{t}^{\text {Cond }}\left(x^{\prime}, A^{\prime}\right)-w_{t}^{\text {Forw }\left[x_{T}, A_{T} ; T\right]}\left(x^{\prime}, A^{\prime} ; x, A\right) \mathcal{P}_{t}^{\text {Cond }}(x, A)\right] \\
& +\sum_{x^{\prime} \neq x} \int d A^{\prime}\left[\mathcal{P}_{t}^{\text {Cond }}\left(x^{\prime}, A^{\prime}\right) w_{t}^{\text {Backw }\left[x_{0}, A_{0} ; 0\right]}\left(x^{\prime}, A^{\prime} ; x, A\right)-\mathcal{P}_{t}^{\text {Cond }}(x, A) w_{t}^{\text {Backw }\left[x_{0}, A_{0} ; 0\right]}\left(x, A ; x^{\prime}, A^{\prime}\right)\right]
\end{aligned}
$$

that is found to vanish using Eqs 4649 and 4. 
(iv) The half-difference between the two dynamical equations of Eqs 45 and 48 yields the new dynamical equation

$$
\begin{aligned}
& \partial_{t} \mathcal{P}_{t}^{\text {Cond }}(x, A)=-\alpha(x) \partial_{A} \mathcal{P}_{t}^{\text {Cond }}(x, A) \\
& +\sum_{x^{\prime} \neq x} \int d A^{\prime}\left[w_{t}^{\left[x_{T}, A_{T} ; T\right],\left[\vec{x}_{0}, A_{0} ; 0\right]}\left(x, A ; x^{\prime}, A^{\prime}\right) \mathcal{P}_{t}^{C o n d}\left(x^{\prime}, A^{\prime}\right)-w_{t}^{\left[x_{T}, A_{T} ; T\right],\left[\vec{x}_{0}, A_{0} ; 0\right]}\left(x^{\prime}, A^{\prime} ; x, A\right) \mathcal{P}_{t}^{\text {Cond }}(x, A)\right]
\end{aligned}
$$

with the time-dependent rates

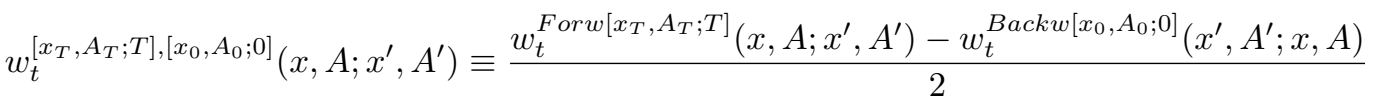

$$
\begin{aligned}
& =\frac{1}{2}\left[P_{T, t}\left(x_{T}, A_{T} \mid x, A\right) w\left(x, A ; x^{\prime}, A^{\prime}\right) \frac{1}{P_{T, t}\left(x_{T}, A_{T} \mid x^{\prime}, A^{\prime}\right)}-\frac{1}{P_{t, 0}\left(x^{\prime}, A^{\prime} \mid x_{0}, A_{0}\right)} w\left(x^{\prime}, A^{\prime} ; x, A\right) P_{t, 0}\left(x, A \mid x_{0}, A_{0}\right)\right]
\end{aligned}
$$

\section{Simple example : conditioning the Sisyphus Markov Jump process on the number of resets}

Let us now consider the continuous-time analog of the Sisyphus Random Walk discussed in subsection III D. The Sisyphus Markov Jump process defined on the half-line $x=0,1,2, \ldots$ is defined as follows : when Sisyphus is at position $x$ at time $t$, he can return to the origin $x=0$ with the reset rate $r$, he can move forward to the next position $(x+1)$ with rate $w$, and otherwise he remains at its position $x$.

As time-additive observable $A$, we will choose the number of resets, so that the joint generator of Eq. 38 becomes

$$
w\left(x, A ; x^{\prime}, A^{\prime}\right) \equiv r \delta_{x, 0} \delta_{A, A^{\prime}+1}+w \delta_{x, x^{\prime}+1} \delta_{A, A^{\prime}} \quad \text { for } \quad(x, A) \neq\left(x^{\prime}, A^{\prime}\right)
$$

\section{Joint propagator $P_{t, t_{0}}\left(x, A \mid x_{0} A_{0}\right)$}

The forward dynamics of Eq. 39 reads for the present model where $A$ is discrete with the generator of Eq. 53

$$
\begin{aligned}
& \partial_{t} P_{t, t_{0}}\left(x, A \mid x_{0}, A_{0}\right)=\sum_{\left(x^{\prime}, A^{\prime}\right) \neq(x, A)}\left[w\left(x, A ; x^{\prime}, A^{\prime}\right) P_{t, t_{0}}\left(x^{\prime}, A^{\prime} \mid x_{0}, A_{0}\right)-w\left(x^{\prime}, A^{\prime} ; x, A\right) P_{t, t_{0}}\left(x, A \mid x_{0}, A_{0}\right)\right] \\
& =r \delta_{x, 0} \theta\left(A>A_{0}\right) \sum_{x^{\prime}=0}^{+\infty} P_{t, t_{0}}\left(x^{\prime}, A-1 \mid x_{0}, A_{0}\right)+w \theta(x>0) P_{t, t_{0}}\left(x-1, A \mid x_{0}, A_{0}\right)-(r+w) P_{t, t_{0}}\left(x, A \mid x_{0}, A_{0}\right)
\end{aligned}
$$

The solution can be directly written from the renewal analysis of the dynamics

$$
\begin{aligned}
& P_{t, t_{0}}\left(x, A \mid x_{0} A_{0}\right)=e^{-r\left(t-t_{0}\right)} \delta_{A, A_{0}} \theta\left(x \geq x_{0}\right) \frac{\left[w\left(t-t_{0}\right)\right]^{\left(x-x_{0}\right)}}{\left(t-t_{0}\right) !} e^{-w\left(t-t_{0}\right)} \\
& +\theta\left(A>A_{0}\right) \theta(x \geq 0) \frac{r^{\left(A-A_{0}\right)}}{\left(A-A_{0}-1\right) !} e^{-r\left(t-t_{0}\right)} \frac{w^{x}}{x !} \int_{0}^{\left(t-t_{0}\right)} d \tau\left[\left(t-t_{0}\right)-\tau\right]^{\left(A-A_{0}\right)-1} \tau^{x} e^{-w \tau}
\end{aligned}
$$

The summation of Eq. 55 over the variable $x$ allows to recover that the propagator for the variable $A$ alone corresponds to the Poisson distribution for $\left(A-A_{0}\right)$ and is independent of $x_{0}$

$$
\sum_{x=0}^{+\infty} P_{t, t_{0}}\left(x, A \mid x_{0} A_{0}\right)=\theta\left(A \geq A_{0}\right) \frac{\left[r\left(t-t_{0}\right)\right]^{\left(A-A_{0}\right)}}{\left(A-A_{0}\right) !} e^{-r\left(t-t_{0}\right)} \equiv P_{t, t_{0}}\left(A \mid A_{0}\right)
$$

The summation of Eq. 55 over the variable $A$ yields the propagator for the initial Markov process $x(t)$ alone

$$
\begin{aligned}
\sum_{A=A_{0}}^{+\infty} P_{t, t_{0}}\left(x, A \mid x_{0} A_{0}\right) & =e^{-r\left(t-t_{0}\right)} \theta\left(x \geq x_{0}\right) \frac{\left[w\left(t-t_{0}\right)\right]^{\left(x-x_{0}\right)}}{\left(t-t_{0}\right) !} e^{-w\left(t-t_{0}\right)}+\theta(x \geq 0) r \frac{w^{x}}{x !} \int_{0}^{\left(t-t_{0}\right)} d \tau \tau^{x} e^{-(w+r) \tau} \\
& \equiv P_{t, t_{0}}\left(x \mid x_{0}\right)
\end{aligned}
$$

that converges towards the steady state corresponding to the geometric distribution

$$
P_{t, t_{0}}\left(x \mid x_{0}\right) \underset{\left(t-t_{0}\right) \rightarrow+\infty}{\simeq} \theta(x \geq 0) r \frac{w^{x}}{x !} \int_{0}^{+\infty} d \tau \tau^{x} e^{-(w+r) \tau}=\frac{r}{w+r}\left(\frac{w}{w+r}\right)^{x} \equiv P_{s t}(x)
$$




\section{Forward generator of the conditioned dynamics}

For the conditioned dynamics, the forward generator of Eq. 46 reads using the joint generator of Eq. 53 for $(x, A) \neq\left(x^{\prime}, A^{\prime}\right)$

$$
\begin{aligned}
w_{t}^{\text {For } w\left[x_{T}, A_{T} ; T\right]}\left(x, A ; x^{\prime}, A^{\prime}\right) & =P_{T, t}\left(x_{T}, A_{T} \mid x, A\right) w\left(x, A ; x^{\prime}, A^{\prime}\right) \frac{1}{P_{T, t}\left(x_{T}, A_{T} \mid x^{\prime}, A^{\prime}\right)} \\
& =\delta_{x, 0} \delta_{A, A^{\prime}+1} r \frac{P_{T, t}\left(x_{T}, A_{T} \mid x, A^{\prime}+1\right)}{P_{T, t}\left(x_{T}, A_{T} \mid x^{\prime}, A^{\prime}\right)}+\delta_{x, x^{\prime}+1} \delta_{A, A^{\prime}} w \frac{P_{T, t}\left(x_{T}, A_{T} \mid x^{\prime}+1, A^{\prime}\right)}{P_{T, t}\left(x_{T}, A_{T} \mid x^{\prime}, A^{\prime}\right)} \\
& \equiv \delta_{x, 0} \delta_{A, A^{\prime}+1} r_{t}^{F o r w\left[x_{T}, A_{T} ; T\right]}\left(x^{\prime}, A^{\prime}\right)+\delta_{x, x^{\prime}+1} \delta_{A, A^{\prime}} w_{t}^{F o r w\left[x_{T}, A_{T} ; T\right]}\left(x^{\prime}, A^{\prime}\right)
\end{aligned}
$$

So the conditioned dynamics corresponds to a Sisyphus Markov jump process, where the initial reset rate $r$ and the initial forward jump rate $w$ have been replaced by reset rates and forward jump rates that depend on the time $t$ and on the configuration $\left(x^{\prime}, A^{\prime}\right)$

$$
\begin{aligned}
r_{t}^{\text {Forw }\left[x_{T}, A_{T} ; T\right]}\left(x^{\prime}, A^{\prime}\right) & =r \frac{P_{T, t}\left(x_{T}, A_{T} \mid x, A^{\prime}+1\right)}{P_{T, t}\left(x_{T}, A_{T} \mid x^{\prime}, A^{\prime}\right)} \\
w_{t}^{\text {For } w\left[x_{T}, A_{T} ; T\right]}\left(x^{\prime}, A^{\prime}\right) & =w \frac{P_{T, t}\left(x_{T}, A_{T} \mid x^{\prime}+1, A^{\prime}\right)}{P_{T, t}\left(x_{T}, A_{T} \mid x^{\prime}, A^{\prime}\right)}
\end{aligned}
$$

where on can plug the explicit form of the joint propagator given in Eq. 55 .

The 'canonical conditioning' (see the reminder in the two Appendices) of the Sisyphus Markov jump process has been studied in [7] for the more general case where the reset rates of the initial model are space-dependent $r_{x}$ (instead of being given by the constant value $r$ ) and where the time-additive observable involve two arbitrary functions $\alpha(x)$ and $\beta\left(x, x^{\prime}\right)$.

\section{APPLICATION TO DIFFUSION PROCESSES IN DIMENSION $d$}

In this section, we consider the diffusion process $\vec{x}(t)$, where the $d$ components $x_{i}(t)$ for $i=1, . ., d$ follow the Langevin stochastic differential equations involving the functions $\left(f_{i}[\vec{x}], g_{i}[\vec{x}]\right)$ and $d$ independent Brownian motions $B_{i}(t)$

$$
d x_{i}(t)=f_{i}[\vec{x}(t)] d t+g_{i}[\vec{x}(t)] d B_{i}(t)
$$

in the Stratonovich interpretation. Equivalently, the dynamics can be defined via the Fokker-Planck equation for the probability $P_{t}(\vec{x})$ to be at position $\vec{x}$ at time $t$

$$
\partial_{t} P_{t}(\vec{x})=\sum_{i=1}^{d} \partial_{x_{i}}\left[-F_{i}[\vec{x}] P_{t}(\vec{x})+D_{i}[\vec{x}] \partial_{x_{i}} P_{t}(\vec{x})\right]
$$

with the following components for the force and for the diffusion coefficient

$$
\begin{aligned}
F_{i}[\vec{x}] & =f_{i}[\vec{x}]-\frac{g_{i}[\vec{x}] \partial_{x_{i}} g_{i}[\vec{x}]}{2} \\
D_{i}[\vec{x}] & =\frac{g_{i}^{2}[\vec{x}]}{2}
\end{aligned}
$$

The time-additive observable $A(t)$ of the trajectory $\vec{x}\left(t_{1} \leq s \leq t_{2}\right)$ of Eq. 1 can be parametrized by the function $\alpha[\vec{x}]$ and by the field $\vec{\beta}[\vec{x}]$ in the Stratonovich interpretation

$$
A\left(t_{2}\right)-A\left(t_{1}\right)=\mathcal{A}\left[x\left(t_{1} \leq s \leq t_{2}\right)\right]=\int_{t_{1}}^{t_{2}}[\alpha[\vec{x}(s)] d s+\vec{\beta}[\vec{x}(s)] \cdot d \vec{x}(s)]
$$




\section{A. Dynamics of the joint propagator $P_{t, t_{0}}\left(\vec{x}, A \mid \vec{x}_{0}, A_{0}\right)$}

Since the increment between $t$ and $(t+d t)$ of the time-additive observable $A(t)$ of Eq. 64 can be rewritten in terms of the $d$ Langevin increments $d x_{i}(t)$ of Eq. 61

$$
\begin{aligned}
d A(t) & =A(t+d t)-A(t)=\alpha[\vec{x}(t)] d t+\sum_{i=1}^{d} \beta_{i}[\vec{x}(t)] \cdot d x_{i}(t) \\
& =\left[\alpha[\vec{x}(t)]+\sum_{i=1}^{d} \beta_{i}[\vec{x}(t)] f_{i}[\vec{x}(t)]\right] d t+\sum_{i=1}^{d} \beta_{i}[\vec{x}(t)] g_{i}[\vec{x}(t)] d B_{i}(t)
\end{aligned}
$$

one can consider that $A(t)$ is a supplementary $(d+1)$ coordinate for the Langevin system in the Stratonovich interpretation of Eq. 61, that involves the $d$ previous Brownian motions $B_{i}(t)$. As a consequence, one can write the Fokker-Planck equations generalizing Eq. 62 as follows :

(i) the forward generator

$$
\mathcal{F}=-\alpha[\vec{x}] \partial_{A}-\sum_{i=1}^{d}\left(\partial_{x_{i}}+\beta_{i}[\vec{x}] \partial_{A}\right) F_{i}[\vec{x}]+\sum_{i=1}^{d}\left(\partial_{x_{i}}+\beta_{i}[\vec{x}] \partial_{A}\right) D_{i}[\vec{x}]\left(\partial_{x_{i}}+\beta_{i}[\vec{x}] \partial_{A}\right)
$$

governs the forward Fokker-Planck equation of the joint propagator $P_{t, 0}\left(\vec{x}, A \mid \vec{x}_{0}, A_{0}\right)$ with respect to the final variables $(\vec{x}, A)$ at time $t$

$$
\begin{aligned}
\partial_{t} P_{t, 0}\left(\vec{x}, A \mid \vec{x}_{0}, A_{0}\right) & =\mathcal{F} P_{t, 0}\left(\vec{x}, A \mid \vec{x}_{0}, A_{0}\right) \\
& =-\alpha[\vec{x}] \partial_{A} P_{t, 0}\left(\vec{x}, A \mid \vec{x}_{0}, A_{0}\right)-\sum_{i=1}^{d}\left(\partial_{x_{i}}+\beta_{i}[\vec{x}] \partial_{A}\right)\left[F_{i}[\vec{x}] P_{t, 0}\left(\vec{x}, A \mid \vec{x}_{0}, A_{0}\right)\right] \\
& +\sum_{i=1}^{d}\left(\partial_{x_{i}}+\beta_{i}[\vec{x}] \partial_{A}\right)\left[D_{i}[\vec{x}]\left(\partial_{x_{i}}+\beta_{i}[\vec{x}] \partial_{A}\right) P_{t, 0}\left(\vec{x}, A \mid \vec{x}_{0}, A_{0}\right)\right]
\end{aligned}
$$

(ii) the backward generator corresponding to the adjoint differential operator of Eq. 66

$$
\mathcal{F}^{\dagger}=\alpha[\vec{x}] \partial_{A}+\sum_{i=1}^{d} F_{i}[\vec{x}]\left(\partial_{x_{i}}+\beta_{i}[\vec{x}] \partial_{A}\right)+\sum_{i=1}^{d}\left(\partial_{x_{i}}+\beta_{i}[\vec{x}] \partial_{A}\right) D_{i}[\vec{x}]\left(\partial_{x_{i}}+\beta_{i}[\vec{x}] \partial_{A}\right)
$$

governs the backward Fokker-Planck equation for the joint propagator $P_{T, t}\left(\vec{x}_{T}, A_{T} \mid \vec{x}, A\right)$ with respect to the initial variables $(\vec{x}, A)$ at time $t$

$$
\begin{aligned}
-\partial_{t} P_{T, t}\left(\vec{x}_{T}, A_{T} \mid \vec{x}, A\right) & =\mathcal{F}^{\dagger} P_{T, t}\left(\vec{x}_{T}, A_{T} \mid \vec{x}, A\right) \\
& =\alpha[\vec{x}] \partial_{A} P_{T, t}\left(\vec{x}_{T}, A_{T} \mid \vec{x}, A\right)+\sum_{i=1}^{d} F_{i}[\vec{x}]\left(\partial_{x_{i}}+\beta_{i}[\vec{x}] \partial_{A}\right) P_{T, t}\left(\vec{x}_{T}, A_{T} \mid \vec{x}, A\right) \\
& +\sum_{i=1}^{d}\left(\partial_{x_{i}}+\beta_{i}[\vec{x}] \partial_{A}\right)\left[D_{i}[\vec{x}]\left(\partial_{x_{i}}+\beta_{i}[\vec{x}] \partial_{A}\right) P_{T, t}\left(\vec{x}_{T}, A_{T} \mid \vec{x}, A\right)\right]
\end{aligned}
$$

B. Markov dynamics for the conditional probability $\mathcal{P}_{t}^{C o n d}(\vec{x}, A)$ with time-dependent additional forces

Let us now focus on the dynamics for the conditional probability of Eq. 4

$$
\mathcal{P}_{t}^{\text {Cond }}(\vec{x}, A)=\frac{P_{T, t}\left(\vec{x}_{T}, A_{T} \mid \vec{x}, A\right) P_{t, 0}\left(\vec{x}, A \mid \vec{x}_{0}, A_{0}\right)}{P_{T, 0}\left(\vec{x}_{T}, A_{T} \mid \vec{x}_{0}, A_{0}\right)}
$$

Its dynamics with respect to the time $t$ involves the forward dynamics with generator $\mathcal{F}$ of Eq. 67 for the propagator $P_{t, 0}\left(\vec{x}, A \mid \vec{x}_{0}, A_{0}\right)$ and the backward dynamics with generator $\mathcal{F}^{\dagger}$ of Eq. 69 for the propagator $P_{T, t}\left(\vec{x}_{T}, A_{T} \mid \vec{x}, A\right)$. So 
the time-derivative of the conditional probability of Eq. 70 reads

$$
\begin{aligned}
\partial_{t} \mathcal{P}_{t}^{C o n d}(\vec{x}, A) & =\left[\partial_{t} P_{T, t}\left(\vec{x}_{T}, A_{T} \mid \vec{x}, A\right)\right] \frac{P_{t, 0}\left(\vec{x}, A \mid \vec{x}_{0}, A_{0}\right)}{P_{T, 0}\left(\vec{x}_{T}, A_{T} \mid \vec{x}_{0}, A_{0}\right)}+\frac{P_{T, t}\left(\vec{x}_{T}, A_{T} \mid \vec{x}, A\right)}{P_{T, 0}\left(\vec{x}_{T}, A_{T} \mid \vec{x}_{0}, A_{0}\right)}\left[\partial_{t} P_{t, 0}\left(\vec{x}, A \mid \vec{x}_{0}, A_{0}\right)\right] \\
& =-\frac{P_{t, 0}\left(\vec{x}, A \mid \vec{x}_{0}, A_{0}\right)}{P_{T, 0}\left(\vec{x}_{T}, A_{T} \mid \vec{x}_{0}, A_{0}\right)}\left[\mathcal{F}^{\dagger} P_{T, t}\left(\vec{x}_{T}, A_{T} \mid \vec{x}, A\right)\right]+\frac{P_{T, t}\left(\vec{x}_{T}, A_{T} \mid \vec{x}, A\right)}{P_{T, 0}\left(\vec{x}_{T}, A_{T} \mid \vec{x}_{0}, A_{0}\right)}\left[\mathcal{F} P_{t, 0}\left(\vec{x}, A \mid \vec{x}_{0}, A_{0}\right)\right]
\end{aligned}
$$

(i) Forward perspective : Eq. 70 allows to plug the propagator

$$
P_{t, 0}\left(\vec{x}, A \mid \vec{x}_{0}, A_{0}\right)=\mathcal{P}_{t}^{C o n d}(\vec{x}, A) \frac{P_{T, 0}\left(\vec{x}_{T}, A_{T} \mid \vec{x}_{0}, A_{0}\right)}{P_{T, t}\left(\vec{x}_{T}, A_{T} \mid \vec{x}, A\right)}
$$

into Eq. 71 to obtain

$$
\partial_{t} \mathcal{P}_{t}^{\text {Cond }}(\vec{x}, A)=-\frac{\mathcal{P}_{t}^{\text {Cond }}(\vec{x}, A)}{P_{T, t}\left(\vec{x}_{T}, A_{T} \mid \vec{x}, A\right)}\left[\mathcal{F}^{\dagger} P_{T, t}\left(\vec{x}_{T}, A_{T} \mid \vec{x}, A\right)\right]+P_{T, t}\left(\vec{x}_{T}, A_{T} \mid \vec{x}, A\right)\left[\mathcal{F} \frac{\mathcal{P}_{t}^{\text {Cond }}(\vec{x}, A)}{P_{T, t}\left(\vec{x}_{T}, A_{T} \mid \vec{x}, A\right)}\right]
$$

or more explicitly using the forms of Eqs 66 and 68 for the differential generator $\mathcal{F}$ and its adjoint $\mathcal{F}^{\dagger}$

$$
\begin{aligned}
\partial_{t} \mathcal{P}_{t}^{C o n d}(\vec{x}, A) & =-\alpha[\vec{x}] \partial_{A} \mathcal{P}_{t}^{C o n d}(\vec{x}, A)-\sum_{i=1}^{d}\left(\partial_{x_{i}}+\beta_{i}[\vec{x}] \partial_{A}\right)\left[\left(F_{i}[\vec{x}]+F_{i}^{F o r w\left[\vec{x}_{T}, A_{T} ; T\right]}[\vec{x}, A ; t]\right) \mathcal{P}_{t}^{C o n d}(\vec{x}, A)\right] \\
& +\sum_{i=1}^{d}\left(\partial_{x_{i}}+\beta_{i}[\vec{x}] \partial_{A}\right)\left[D_{i}[\vec{x}]\left(\partial_{x_{i}}+\beta_{i}[\vec{x}] \partial_{A}\right) \mathcal{P}_{t}^{C o n d}(\vec{x}, A)\right]
\end{aligned}
$$

where the only differences with respect to the forward joint Fokker-Planck dynamics of Eq. 67 are the additional time-dependent forces

$$
F_{i}^{F o r w\left[\vec{x}_{T}, A_{T} ; T\right]}[\vec{x}, A ; t] \equiv 2 D_{i}[\vec{x}]\left(\partial_{x_{i}}+\beta_{i}[\vec{x}] \partial_{A}\right) \ln P_{T, t}\left(\vec{x}_{T}, A_{T} \mid \vec{x}, A\right)
$$

that involve the propagator $P_{T, t}\left(\vec{x}_{T}, A_{T} \mid \vec{x}, A\right)$ up to the imposed final values $\left(\vec{x}_{T}, A_{T}\right)$ at time $T$. Eq. 75 is the analog of Eqs 18 and 46.

(ii) Backward perspective : Eq. 70 allows to plug the propagator

$$
P_{T, t}\left(\vec{x}_{T}, A_{T} \mid \vec{x}, A\right)=\mathcal{P}_{t}^{C o n d}(\vec{x}, A) \frac{P_{T, 0}\left(\vec{x}_{T}, A_{T} \mid \vec{x}_{0}, A_{0}\right)}{P_{t, 0}\left(\vec{x}, A \mid \vec{x}_{0}, A_{0}\right)}
$$

into Eq. 71 to obtain

$$
-\partial_{t} \mathcal{P}_{t}^{\text {Cond }}(\vec{x}, A)=P_{t, 0}\left(\vec{x}, A \mid \vec{x}_{0}, A_{0}\right)\left[\mathcal{F}^{\dagger} \frac{\mathcal{P}_{t}^{C o n d}(\vec{x}, A)}{P_{t, 0}\left(\vec{x}, A \mid \vec{x}_{0}, A_{0}\right)}\right]-\frac{\mathcal{P}_{t}^{C o n d}(\vec{x}, A)}{P_{t, 0}\left(\vec{x}, A \mid \vec{x}_{0}, A_{0}\right)}\left[\mathcal{F} P_{t, 0}\left(\vec{x}, A \mid \vec{x}_{0}, A_{0}\right)\right]
$$

or more explicitly using the forms of Eqs 66 and 68 for the differential generator $\mathcal{F}$ and its adjoint $\mathcal{F}^{\dagger}$

$$
\begin{aligned}
-\partial_{t} \mathcal{P}_{t}^{\text {Cond }}(\vec{x}, A) & =\alpha[\vec{x}] \partial_{A} \mathcal{P}_{t}^{C o n d}(\vec{x}, A)+\sum_{i=1}^{d}\left(\partial_{x_{i}}+\beta_{i}[\vec{x}] \partial_{A}\right)\left[\left(F_{i}[\vec{x}]+F_{i}^{\text {Backw }\left[\vec{x}_{0}, A_{0} ; 0\right]}[\vec{x}, A ; t]\right) \mathcal{P}_{t}^{C o n d}(\vec{x}, A)\right] \\
& +\sum_{i=1}^{d}\left(\partial_{x_{i}}+\beta_{i}[\vec{x}] \partial_{A}\right)\left[D_{i}[\vec{x}]\left(\partial_{x_{i}}+\beta_{i}[\vec{x}] \partial_{A}\right) \mathcal{P}_{t}^{\text {Cond }}(\vec{x}, A)\right]
\end{aligned}
$$

where the time-dependent forces

$$
F_{i}^{B a c k w\left[\vec{x}_{0}, A_{0} ; 0\right]}[\vec{x}, A ; t] \equiv-2 D_{i}[\vec{x}]\left(\partial_{x_{i}}+\beta_{i}[\vec{x}] \partial_{A}\right) \ln P_{t, 0}\left(\vec{x}, A \mid \vec{x}_{0}, A_{0}\right)
$$

involve the propagator $P_{t, 0}\left(\vec{x}, A \mid \vec{x}_{0}, A_{0}\right)$ up to the imposed initial values $\left(\vec{x}_{0}, A_{0}\right)$ at time $t=0$ Eq. 79 is the analog of Eqs 23 and 49 . 
(iii) The compatibility between the two dynamical equations of Eqs 74 and 77 can be checked via their sum that can be evaluated using the explicit expressions of Eqs 75 and 79 for the additional forces

$$
\begin{aligned}
0 & \stackrel{?}{=} \sum_{i=1}^{d}\left(\partial_{x_{i}}+\beta_{i}[\vec{x}] \partial_{A}\right)\left[\left(F_{i}^{\text {Backw }\left[\vec{x}_{0}, A_{0} ; 0\right]}[\vec{x}, A ; t]-F_{i}^{F o r w\left[\vec{x}_{T}, A_{T} ; T\right]}[\vec{x}, A ; t]\right) \mathcal{P}_{t}^{\text {Cond }}(\vec{x}, A)+2 D_{i}[\vec{x}]\left(\partial_{x_{i}}+\beta_{i}[\vec{x}] \partial_{A}\right) \mathcal{P}_{t}^{\text {Cond }}(\vec{x}, A)\right] \\
& =\sum_{i=1}^{d}\left(\partial_{x_{i}}+\beta_{i}[\vec{x}] \partial_{A}\right) 2 D_{i}[\vec{x}] \mathcal{P}_{t}^{\text {Cond }}(\vec{x}, A)\left[\left(\partial_{x_{i}}+\beta_{i}[\vec{x}] \partial_{A}\right) \ln \frac{\mathcal{P}_{t}^{\text {Cond }}(\vec{x}, A)}{P_{T, t}\left(\vec{x}_{T}, A_{T} \mid \vec{x}, A\right) P_{t, 0}\left(\vec{x}, A \mid \vec{x}_{0}, A_{0}\right)}\right] \\
& =\sum_{i=1}^{d}\left(\partial_{x_{i}}+\beta_{i}[\vec{x}] \partial_{A}\right) 2 D_{i}[\vec{x}] \mathcal{P}_{t}^{\text {Cond }}(\vec{x}, A)\left[\left(\partial_{x_{i}}+\beta_{i}[\vec{x}] \partial_{A}\right) \ln \frac{1}{P_{T, 0}\left(\vec{x}_{T}, A_{T} \mid \vec{x}_{0}, A_{0}\right)}\right]=0
\end{aligned}
$$

where we have used Eq. 70 to obtain the propagator $P_{T, 0}\left(\vec{x}_{T}, A_{T} \mid \vec{x}_{0}, A_{0}\right)$ that does not depend upon $(\vec{x}, A)$.

(iv) The half-difference between the two dynamical equations of Eqs 74 and 77 leads to the new dynamical equation involving only drift contributions

$$
\partial_{t} \mathcal{P}_{t}^{\text {Cond }}(\vec{x}, A)=-\alpha[\vec{x}] \partial_{A} \mathcal{P}_{t}^{\text {Cond }}(\vec{x}, A)-\sum_{i=1}^{d}\left(\partial_{x_{i}}+\beta_{i}[\vec{x}] \partial_{A}\right)\left[\left(F_{i}[\vec{x}]+F_{i}^{\left[\vec{x}_{T}, A_{T} ; T\right],\left[\vec{x}_{0}, A_{0} ; 0\right]}[\vec{x}, A ; t]\right) \mathcal{P}_{t}^{\text {Cond }}(\vec{x}, A)\right]
$$

where the time-dependent additional forces

$$
\begin{aligned}
F_{i}^{\left[\vec{x}_{T}, A_{T} ; T\right],\left[\vec{x}_{0}, A_{0} ; 0\right]}[\vec{x}, A ; t] & \equiv \frac{F_{i}^{F o r w\left[\vec{x}_{T}, A_{T} ; T\right]}[\vec{x}, A ; t]+F_{i}^{\text {Backw }\left[\vec{x}_{0}, A_{0} ; 0\right]}[\vec{x}, A ; t]}{2} \\
& =D_{i}[\vec{x}]\left(\partial_{x_{i}}+\beta_{i}[\vec{x}] \partial_{A}\right) \ln \frac{P_{T, t}\left(\vec{x}_{T}, A_{T} \mid \vec{x}, A\right)}{P_{t, 0}\left(\vec{x}, A \mid \vec{x}_{0}, A_{0}\right)}
\end{aligned}
$$

involves both propagators $P_{t, 0}\left(\vec{x}, A \mid \vec{x}_{0}, A_{0}\right)$ and $P_{T, t}\left(\vec{x}_{T}, A_{T} \mid \vec{x}, A\right)$.

\section{Stratonovich stochastic differential equations for the conditioned process $\left(\vec{x}^{*}(t), A^{*}(t)\right)$}

The forward Fokker-Planck dynamics of Eq. 74 can be translated into the following Stratonovich stochastic differential equations for the joint conditioned process $\left(\vec{x}^{*}(t), A^{*}(t)\right)$. The $d$ components $x_{i}^{*}(t)$ for $i=1, . ., d$ of $\vec{x}^{*}(t)$ satisfy the Stratonovich stochastic differential equations in terms of $d$ independent Brownian motions $B_{i}(t)$

$$
d x_{i}^{*}(t)=\left(f_{i}\left[\vec{x}^{*}(t)\right]+F_{i}^{F o r w ~}\left[\vec{x}_{T}, A_{T} ; T\right]\left[\vec{x}^{*}(t), A^{*}(t) ; t\right]\right) d t+g_{i}\left[\vec{x}^{*}(t)\right] d B_{i}(t)
$$

where the only differences with respect to the unconditioned case of Eq. 61 are the additional time-dependent forces $F_{i}^{F o r w\left[\vec{x}_{T}, A_{T} ; T\right]}\left[\vec{x}^{*}, A^{*} ; t\right]$ given in Eq. 75 .

Since the increment between $t$ and $(t+d t)$ of the time-additive observable $A^{*}(t)$ can be rewritten in terms of $\vec{x}^{*}(t)$ and of the $d$ Langevin increments $d x_{i}^{*}(t)$ of Eq. 83, the Stratonovich stochastic differential equation for $A^{*}(t)$ reads

$$
\begin{aligned}
& d A^{*}(t)=A^{*}(t+d t)-A^{*}(t)=\alpha\left[\vec{x}^{*}(t)\right] d t+\sum_{i=1}^{d} \beta_{i}\left[\vec{x}^{*}(t)\right] . d x_{i}^{*}(t) \\
& =\left[\alpha\left[\vec{x}^{*}(t)\right]+\sum_{i=1}^{d} \beta_{i}\left[\vec{x}^{*}(t)\right]\left(f_{i}\left[\vec{x}^{*}(t)\right]+F_{i}^{F o r w\left[\vec{x}_{T}, A_{T} ; T\right]}\left[\vec{x}^{*}(t), A^{*}(t) ; t\right]\right)\right] d t+\sum_{i=1}^{d} \beta_{i}\left[\vec{x}^{*}(t)\right] g_{i}\left[\vec{x}^{*}(t)\right] d B_{i}(t)
\end{aligned}
$$

The Stratonovich Stochastic Differential Equations of Eqs 83 and 84 can be then used to generate stochastic trajectories of the conditioned process $\left(\vec{x}^{*}(t), A^{*}(t)\right)$.

\section{Simple example : Brownian $B(t)$ as time-additive observable of the diffusion process $x(t)$}

1. Brownian motion $B(t)$ conditioned on the value of the diffusion process $x(t)$

Let us consider the one-dimensional diffusion process of Eq. 61

$$
d x(t)=f[x(t)] d t+g[x(t)] d B(t)
$$


associated to the Fokker-Planck Eq. 62

$$
\partial_{t} P_{t}(x)=\partial_{x}\left[-F[x] P_{t}(x)+D[x] \partial_{x} P_{t}(x)\right]
$$

with the the force and the diffusion coefficient of Eq. 63

$$
\begin{aligned}
F[x] & =f[x]-\frac{g[x] g^{\prime}[x]}{2} \\
D[x] & =\frac{g^{2}[x]}{2}
\end{aligned}
$$

As time-additive observable, let us choose the Brownian motion $B(t)$ satisfying Eq. 85

$$
d B(t)=-\frac{f[x(t)]}{g[x(t)]} d t+\frac{1}{g[x(t)]} d x(t)
$$

i.e. the two functions $\alpha$ and $\beta$ are present in the parametrization of Eq. 64

$$
\begin{aligned}
\alpha[x] & =-\frac{f[x]}{g[x]} \\
\beta[x] & =\frac{1}{g[x]}
\end{aligned}
$$

The joint propagator $P_{t, t_{0}}\left(x, B \mid x_{0}, B_{0}\right)$ satisfies the forward dynamics of Eq. 67

$$
\begin{aligned}
\partial_{t} P_{t, t_{0}}\left(x, B \mid x_{0}, B_{0}\right) & =\partial_{x}\left[-F[x] P_{t, t_{0}}\left(x, B \mid x_{0}, B_{0}\right)+D[x] \partial_{x} P_{t, t_{0}}\left(x, B \mid x_{0}, B_{0}\right)+\sqrt{2 D[x]} \partial_{B} P_{t, t_{0}}\left(x, B \mid x_{0}, B_{0}\right)\right] \\
& +\frac{1}{2} \partial_{B}^{2} P_{t, t_{0}}\left(x, B \mid x_{0}, B_{0}\right)
\end{aligned}
$$

Since in the microcanonical conditioning framework one considers the joint process $(x(t), B(t))$, one can rephrase the 'conditioning of the diffusion process $x(t)$ on its time-additive observable $B(t)$ ' described above as the 'conditioning of Brownian motion $B(t)$ on the diffusion process $x(t)^{\prime}$ '. This rephrasing is interesting because the diffusion process $x(t)$ generated via Eq. 85 is not a time-additive observable of the Brownian motion $B(t)$.

When the joint propagator $P_{t, t_{0}}\left(x, B \mid x_{0}, B_{0}\right)$ of Eq. 90 is explicit, one can compute the additional time-dependent force of Eq. 75

$$
F^{F o r w\left[x_{T}, B_{T} ; T\right]}[x, B ; t]=2 D[x]\left(\partial_{x}+\beta[x] \partial_{B}\right) \ln P_{T, t}\left(x_{T}, B_{T} \mid x, B\right)
$$

that appear in the Stratonovich stochastic differential equations for the conditioned process $\left(x^{*}(t), B^{*}(t)\right)$ as follows. The Stratonovich stochastic differential equation of Eq 83 for $x^{*}(t)$ involves a Wiener process $W(t)$

$$
d x^{*}(t)=\left(f\left[x^{*}(t)\right]+F^{F o r w\left[\vec{x}_{T}, B_{T} ; T\right]}\left[x^{*}(t), B^{*}(t) ; t\right]\right) d t+g\left[x^{*}(t)\right] d W(t)
$$

while the Stratonovich stochastic differential equation of Eq. 84 for $B^{*}(t)$ reads using Eqs 88 and 89

$$
\begin{aligned}
d B^{*}(t) & =\frac{\left[-f\left[x^{*}(t)\right] d t+d x^{*}(t)\right]}{g\left[x^{*}(t)\right]} \\
& =\frac{F^{F o r w\left[\vec{x}_{T}, B_{T} ; T\right]}\left[x^{*}(t), B^{*}(t) ; t\right]}{g\left[x^{*}(t)\right]} d t+d W(t)
\end{aligned}
$$

The dynamics of Eq. 92 can also be rewritten in terms of the increment $d B^{*}(t)$ of Eq. 93 as

$$
d x^{*}(t)=f\left[x^{*}(t)\right] d t+g\left[x^{*}(t)\right] d B^{*}(t)
$$




\section{Explicit solution when $x(t)$ is the Ornstein-Uhlenbeck process}

In order to have a simple Gaussian solution for the joint propagator, let us now consider the case of the OrnsteinUhlenbeck process for $x(t)$ corresponding to the constant diffusion coefficient and the linear restoring force

$$
\begin{aligned}
& D[x]=D \\
& F[x]=-x
\end{aligned}
$$

so that the corresponding stochastic differential equation reads

$$
d x(t)=-x(t) d t+\sqrt{2 D} d B(t)
$$

both in the Stratonovich and in the Ito interpretations, since the diffusion constant is space-independent.

The forward Fokker-planck Eq. 90 for the joint propagator $P_{t, t_{0}}\left(x, B \mid x_{0}, B_{0}\right)$

$$
\begin{aligned}
\partial_{t} P_{t, t_{0}}\left(x, B \mid x_{0}, B_{0}\right) & =\partial_{x}\left[x P_{t, t_{0}}\left(x, B \mid x_{0}, B_{0}\right)\right]+D \partial_{x}^{2} P_{t, t_{0}}\left(x, B \mid x_{0}, B_{0}\right)+\sqrt{2 D} \partial_{B} \partial_{x} P_{t, t_{0}}\left(x, B \mid x_{0}, B_{0}\right) \\
& +\frac{1}{2} \partial_{B}^{2} P_{t, t_{0}}\left(x, B \mid x_{0}, B_{0}\right)
\end{aligned}
$$

can be translated via the double Fourier transform

$$
\hat{P}_{t, t_{0}}\left(q, k \mid x_{0}, B_{0}\right) \equiv \int_{-\infty}^{+\infty} d x e^{i q x} \int_{-\infty}^{+\infty} d B e^{i k B} P_{t, t_{0}}\left(x, B \mid x_{0}, B_{0}\right)
$$

into the dynamical equation

$$
\partial_{t} \hat{P}_{t, t_{0}}\left(q, k \mid x_{0}, B_{0}\right)=-q \partial_{q} \hat{P}_{t, t_{0}}\left(q, k \mid x_{0}, B_{0}\right)-\left[D q^{2}+\sqrt{2 D} q k+\frac{k^{2}}{2}\right] \hat{P}_{t, t_{0}}\left(q, k \mid x_{0}, B_{0}\right)
$$

and the initial conditions at $t=t_{0}$

$$
\hat{P}_{t=t_{0}, t_{0}}\left(q, k \mid x_{0}, B_{0}\right) \equiv \int_{-\infty}^{+\infty} d x e^{i q x} \int_{-\infty}^{+\infty} d B e^{i k B} \delta\left(x-x_{0}\right) \delta\left(B-B_{0}\right)=e^{i q x_{0}} e^{i k B_{0}}
$$

The solution

$$
\hat{P}_{t, t_{0}}\left(q, k \mid x_{0}, B_{0}\right)=e^{-q^{2} \frac{D}{2}\left[1-e^{-2\left(t-t_{0}\right)}\right]-k^{2} \frac{t-t_{0}}{2}-k q \sqrt{2 D}\left[1-e^{-\left(t-t_{0}\right)}\right]+i q x_{0} e^{-\left(t-t_{0}\right)}+i k B_{0}}
$$

corresponds via the double-inverse Fourier transform of Eq. 98 to the bivariate Gaussian distribution

$$
\begin{aligned}
& P_{t, t_{0}}\left(x, B \mid x_{0}, B_{0}\right)=\int_{-\infty}^{+\infty} \frac{d q}{2 \pi} e^{-i q x} \int_{-\infty}^{+\infty} \frac{d k}{2 \pi} e^{-i k B} \hat{P}_{t, t_{0}}\left(q, k \mid x_{0}, B_{0}\right) \\
& =\frac{1}{2 \pi \sigma\left(t, t_{0}\right) v\left(t, t_{0}\right) \sqrt{1-c^{2}\left(t, t_{0}\right)}} e^{-\frac{1}{2\left[1-c^{2}\left(t, t_{0}\right)\right]}\left[\left(\frac{x-x_{0} e^{-\left(t-t_{0}\right)}}{\sigma\left(t, t_{0}\right)}\right)^{2}+\left(\frac{B-B_{0}}{v\left(t, t_{0}\right)}\right)^{2}-2 c\left(t, t_{0}\right)\left(\frac{x-x_{0} e^{-\left(t-t_{0}\right)}}{\sigma\left(t, t_{0}\right)}\right)\left(\frac{B-B_{0}}{v\left(t, t_{0}\right)}\right)\right]}
\end{aligned}
$$

with the two variances

$$
\begin{aligned}
\sigma^{2}\left(t, t_{0}\right) & =D\left[1-e^{-2\left(t-t_{0}\right)}\right] \\
v^{2}\left(t, t_{0}\right) & =\left(t-t_{0}\right)
\end{aligned}
$$

and the rescaled correlation

$$
c\left(t, t_{0}\right)=\sqrt{\frac{2\left[1-e^{-\left(t-t_{0}\right)}\right]}{\left(t-t_{0}\right)\left[1+e^{-\left(t-t_{0}\right)}\right]}}
$$

The conditioned forward dynamics is then governed by the Fokker-Planck Eq. 74

$$
\begin{aligned}
& \partial_{t} \mathcal{P}_{t}^{C o n d}(x, B)=-\partial_{x}\left[\left(-x+F_{t}^{F o r w ~}\left[x_{T}, B_{T} ; T\right][x, B]\right) P_{t, t_{0}}\left(x, B \mid x_{0}, B_{0}\right)\right] \\
& +D \partial_{x}^{2} P_{t, t_{0}}\left(x, B \mid x_{0}, B_{0}\right)+\sqrt{2 D} \partial_{B} \partial_{x} P_{t, t_{0}}\left(x, B \mid x_{0}, B_{0}\right)+\frac{1}{2} \partial_{B}^{2} P_{t, t_{0}}\left(x, B \mid x_{0}, B_{0}\right)
\end{aligned}
$$


where the only difference with respect to the forward Fokker-Planck dynamics of Eq. 97 is the additional timedependent force of Eq. 75

$$
F_{t}^{F o r w\left[x_{T}, B_{T} ; T\right]}[x, B] \equiv 2 D\left(\partial_{x}+\frac{1}{\sqrt{2 D}} \partial_{B}\right) \ln P_{T, t}\left(x_{T}, B_{T} \mid x, B\right)
$$

Using the explicit form of Eq. 102 for the propagator, one obtains

$$
\begin{aligned}
& \ln P_{T, t}\left(x_{T}, B_{T} \mid x, B\right)=-\ln \left(2 \pi \sigma(T, t) v(T, t) \sqrt{1-c^{2}(T, t)}\right) \\
& -\frac{1}{2\left[1-c^{2}(T, t)\right]}\left[\left(\frac{x e^{-(T-t)}-x_{T}}{\sigma(T, t)}\right)^{2}+\left(\frac{B-B_{T}}{v(T, t)}\right)^{2}-2 c(T, t)\left(\frac{x e^{-(T-t)}-x_{T}}{\sigma(T, t)}\right)\left(\frac{B-B_{T}}{v(T, t)}\right)\right]
\end{aligned}
$$

with the corresponding partial derivatives with respect to $x$

$$
\partial_{x} \ln P_{T, t}\left(x_{T}, B_{T} \mid x, B\right)=\frac{e^{-(T-t)}}{\sigma(T, t)\left[1-c^{2}(T, t)\right]}\left[-\left(\frac{x e^{-(T-t)}-x_{T}}{\sigma(T, t)}\right)+c(T, t)\left(\frac{B-B_{T}}{v(T, t)}\right)\right]
$$

and with respect to $B$

$$
\partial_{B} \ln P_{T, t}\left(x_{T}, B_{T} \mid x, B\right)=\frac{1}{v(T, t)\left[1-c^{2}(T, t)\right]}\left[-\left(\frac{B-B_{T}}{v(T, t)}\right)+c(T, t)\left(\frac{x e^{-(T-t)}-x_{T}}{\sigma(T, t)}\right)\right]
$$

As a consequence, the additional time-dependent force of Eq. 106 is linear with respect to $x$ and with respect to $B$

$$
\begin{aligned}
& F_{t}^{F o r w\left[x_{T}, B_{T} ; T\right]}[x, B]=\left[\sqrt{2 D} \partial_{x} \ln P_{T, t}\left(x_{T}, B_{T} \mid x, B\right)+\partial_{B} \ln P_{T, t}\left(x_{T}, B_{T} \mid x, B\right)\right] \\
& =\frac{\sqrt{2 D}}{\left[1-c^{2}(T, t)\right]}\left[\frac{c(T, t)}{v(T, t)}-\frac{\sqrt{2 D} e^{-(T-t)}}{\sigma(T, t)}\right]\left(\frac{x e^{-(T-t)}-x_{T}}{\sigma(T, t)}\right) \\
& +\frac{\sqrt{2 D}}{\left[1-c^{2}(T, t)\right]}\left[\frac{\sqrt{2 D} c(T, t) e^{-(T-t)}}{\sigma(T, t)}-\frac{1}{v(T, t)}\right]\left(\frac{B-B_{T}}{v(T, t)}\right)
\end{aligned}
$$

while the time-dependence is governed by the functions introduced in Eqs 103 and 104

$$
\begin{aligned}
\sigma(T, t) & =\sqrt{D\left[1-e^{-2(T-t)}\right]} \\
v(T, t) & =\sqrt{T-t} \\
c(T, t) & =\sqrt{\frac{2\left[1-e^{-(T-t)}\right]}{(T-t)\left[1+e^{-(T-t)}\right]}}
\end{aligned}
$$

The explicit time-dependent force $F_{t}^{F o r w\left[x_{T}, B_{T} ; T\right]}[x, B]$ of Eq. 110 can be then plugged into the stochastic differential equation of Eq. 93 involving a Wiener process $W(t)$

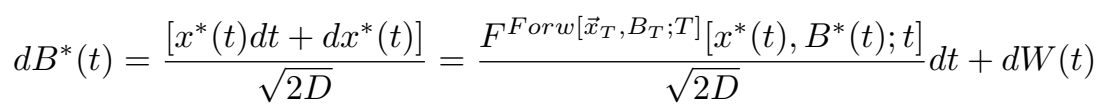

while the stochastic differential equation for $x^{*}(t)$ can be written either as Eq. 92

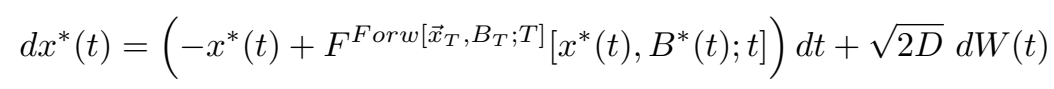

or as Eq. 94

$$
d x^{*}(t)=-x^{*}(t) d t+\sqrt{2 D} d B^{*}(t)
$$

in order to generate stochastic trajectories of the conditioned process $\left(x^{*}(t), B^{*}(t)\right)$.

As a final remark, let us stress that other explicit examples of microcanonical conditioning for the Brownian motion or the Ornstein-Uhlenbeck process on various time-additive observables can be found in $[75,76,80]$. 


\section{CONCLUSION}

In this paper, the recent studies concerning the conditioning of one-dimensional diffusion processes or discrete-time random walks on global dynamical constraints over a finite time-window $T[75,76,80]$ have been generalized to analyze the 'microcanonical conditioning' of Markov processes on time-additive observables. We have described the application to various types of Markov processes, namely discrete-time Markov chains, continuous-time Markov jump processes and diffusion processes in arbitrary dimension. In each setting, we have considered the most general time-additive observable that can involve both the time spent in each configuration and the elementary increments of the Markov process. We have illustrated the various cases via simple explicit examples. In the two Appendices, we describe the link with the 'canonical conditioning' based on the generating function of the time-additive observable, that has been much studied recently in the field of non-equilibrium steady states $[16,31-74]$ as recalled in the Introduction.

We hope that the present general formulation of the 'microcanonical conditioning' of Markov processes on timeadditive observables will be helpful to identity new soluble cases besides the various explicit solutions given in the recent works $[75,76,80]$.

\section{Appendix A: Links with the canonical conditioning on a time-additive observable for finite time $T$}

As recalled in more details in the Introduction, the 'canonical conditioning' of Markov processes has been much studied recently in the field of non-equilibrium steady states [16, 31-74]. In this Appendix, it is thus interesting to describe the links with the microcanonical conditioning considered in the main text.

\section{Generating function $Z_{t, t_{0}}^{[k]}\left(x \mid x_{0}\right)$ of the total increment $A(t)-A\left(t_{0}\right)=\mathcal{A}\left[x\left(t_{0} \leq s \leq t\right)\right]$}

Here the basic object is the generating function $Z_{t, t_{0}}^{[k]}\left(x \mid x_{0}\right)$ of the total increment $A(t)-A\left(t_{0}\right)=\mathcal{A}\left[x\left(t_{0} \leq s \leq t\right)\right]$ over the Markov trajectories $x\left(t_{0} \leq s \leq t\right)$ starting at $x\left(t_{0}\right)=x_{0}$ and ending at $x(t)=x$

$$
Z_{t, t_{0}}^{[k]}\left(x \mid x_{0}\right) \equiv\left\langle\delta_{x(t), x} e^{k \mathcal{A}\left[x\left(t_{0} \leq s \leq t\right)\right]} \delta_{x\left(t_{0}\right), x_{0}}\right\rangle
$$

For fixed $k$, the generating function $Z_{t, t_{0}}^{[k]}\left(x \mid x_{0}\right)$ satisfies

(i) some forward $k$-dependent dynamics with respect to the final state $x$ at time $t$, that can be obtained from the forward dynamics of the joint propagator $P_{t, t_{0}}\left(x, A \mid x_{0}, A_{0}\right)$ of Eq. 2 via

$$
Z_{t, t_{0}}^{[k]}\left(x \mid x_{0}\right)=\sum_{A} e^{k A} P_{t, t_{0}}\left(x, A \mid x_{0}, A_{0}=0\right)
$$

(ii) some backward $k$-dependent dynamics with respect to the initial state $x_{0}$ at time $t_{0}$, that can be obtained from the backward dynamics of the joint propagator $P_{t, t_{0}}\left(x, A \mid x_{0}, A_{0}\right)$ of Eq. 2 via

$$
Z_{t, t_{0}}^{[k]}\left(x \mid x_{0}\right)=\sum_{A_{0}} e^{-k A_{0}} P_{t, t_{0}}\left(x, A=0 \mid x_{0}, A_{0}\right)
$$

Here it is important to stress that these two dynamics are not probability-conserving Markov dynamics, since $Z_{t, t_{0}}^{[k]}\left(x \mid x_{0}\right)$ is a generating function and not a probability.

a. Dynamics of the generating function $Z_{t, t_{0}}^{[k]}\left(x \mid x_{0}\right)$ for discrete-time Markov chains of section III

The joint generator $W\left(x, A ; x^{\prime}, A^{\prime}\right)$ of Eq. 11 is in correspondence with the $k$-tilted matrix

$$
W^{[k]}\left(x ; x^{\prime}\right)=\sum_{A} W\left(x, A ; x^{\prime}, A^{\prime}\right) e^{k\left(A-A^{\prime}\right)}=W\left(x ; x^{\prime}\right) e^{k \beta\left(x, x^{\prime}\right)}
$$

(i) The forward dynamics of Eq. 13 for the joint propagator $P_{t, t_{0}}\left(x, A \mid x_{0}, A_{0}\right)$ translates into the following forward dynamics for the generating function $Z_{t, t_{0}}^{[k]}\left(x \mid x_{0}\right)$ via Eq. A2

$$
Z_{t+1, t_{0}}^{[k]}\left(x \mid x_{0}\right)=\sum_{x^{\prime}} W^{[k]}\left(x ; x^{\prime}\right) Z_{t, t_{0}}^{[k]}\left(x^{\prime} \mid x_{0}\right)
$$


(ii) The backward dynamics of Eq. 14 for the joint propagator $P_{t, t_{0}}\left(x, A \mid x_{0}, A_{0}\right)$ translates into the following backward dynamics for the generating function via Eq. A3

$$
Z_{t, t_{0}-1}^{[k]}\left(x \mid x_{0}\right)=\sum_{x_{0}^{\prime}} Z_{t, t_{0}}\left(x \mid x_{0}^{\prime}\right) W^{[k]}\left(x_{0}^{\prime} ; x_{0}\right)
$$

b. Dynamics of the generating function $Z_{t, t_{0}}^{[k]}\left(x \mid x_{0}\right)$ for continuous-time Markov jump processes of section IV

The jump-drift dynamics of Eqs 36 and 38 is in correspondence with the $k$-tilted matrix

$$
\begin{aligned}
w^{[k]}(x ; x) & \equiv k \alpha(x)-\sum_{x^{\prime} \neq x} w\left(x^{\prime} ; x\right) \\
w^{[k]}\left(x ; x^{\prime}\right) & \equiv w\left(x ; x^{\prime}\right) e^{k \beta\left(x, x^{\prime}\right)} \quad \text { for } x \neq x^{\prime}
\end{aligned}
$$

(i) The forward dynamics of Eq. 39 for the joint propagator $P_{t, t_{0}}\left(x, A \mid x_{0}, A_{0}\right)$ translates into the following forward dynamics for the generating function $Z_{t, t_{0}}^{[k]}\left(x \mid x_{0}\right)$ via Eq. A2

$$
\partial_{t} Z_{t, t_{0}}^{[k]}\left(x \mid x_{0}\right)=\sum_{x^{\prime}} w^{[k]}\left(x ; x^{\prime}\right) Z_{t, t_{0}}^{[k]}\left(x^{\prime} \mid x_{0}\right)
$$

(ii) The backward dynamics of Eq. 40 for the joint propagator $P_{t, t_{0}}\left(x, A \mid x_{0}, A_{0}\right)$ translates into the following backward dynamics for the generating function via Eq. A3

$$
\begin{aligned}
& \qquad-\partial_{t_{0}} Z_{t, t_{0}}^{[k]}\left(x \mid x_{0}\right)=\sum_{x_{0}^{\prime}} Z_{t, t_{0}}^{[k]}\left(x \mid x_{0}^{\prime}\right) w^{[k]}\left(x_{0}^{\prime} ; x_{0}\right) \\
& \text { c. Dynamics of the generating function } Z_{t, t_{0}}^{[k]}\left(\vec{x} \mid \vec{x}_{0}\right) \text { for diffusion processes of section } V
\end{aligned}
$$

(i) The forward generator of Eq. 66 corresponds to the $k$-tilted differential operator

$$
\mathcal{F}_{k}=k \alpha[\vec{x}]-\sum_{i=1}^{d}\left(\partial_{i}-k \beta_{i}[\vec{x}]\right) F_{i}[\vec{x}]+\sum_{i=1}^{d}\left(\partial_{i}-k \beta_{i}[\vec{x}]\right) D_{i}[\vec{x}]\left(\partial_{i}-k \beta_{i}[\vec{x}]\right)
$$

The forward dynamics of Eq. 67 for the joint propagator $P_{t, t_{0}}\left(\vec{x}, A \mid \vec{x}_{0}, A_{0}\right)$ translates for the generating function via Eq. A2 into the forward dynamics

$$
\begin{aligned}
\partial_{t} Z_{t, 0}^{[k]}\left(\vec{x} \mid \vec{x}_{0}\right) & \equiv \mathcal{F}_{k} Z_{t, 0}^{[k]}\left(\vec{x} \mid \vec{x}_{0}\right) \\
& =k \alpha[\vec{x}] Z_{t, 0}^{[k]}\left(\vec{x} \mid \vec{x}_{0}\right)-\sum_{i=1}^{d}\left(\partial_{i}-k \beta_{i}[\vec{x}]\right)\left[F_{i}[\vec{x}] Z_{t, 0}^{[k]}\left(\vec{x} \mid \vec{x}_{0}\right)\right] \\
& +\sum_{i=1}^{d}\left(\partial_{i}-k \beta_{i}[\vec{x}]\right)\left[D_{i}[\vec{x}]\left(\partial_{i}-k \beta_{i}[\vec{x}]\right)\left(Z_{t, 0}^{[k]}\left(\vec{x} \mid \vec{x}_{0}\right)\right)\right]
\end{aligned}
$$

(ii) The backward dynamics of Eq. 69 for the joint propagator $P_{T, t}\left(\vec{x}_{T}, A_{T} \mid \vec{x}, A\right)$ can be translated for the generating function via Eq. A3 into the backward dynamics

$$
\begin{aligned}
-\partial_{t} Z_{T, t}^{[k]}\left(\vec{x}_{T} \mid \vec{x}\right) & =k \alpha[\vec{x}] Z_{T, t}^{[k]}\left(\vec{x}_{T} \mid \vec{x}\right)+\sum_{i=1}^{d} F_{i}[\vec{x}]\left(\partial_{i}+k \beta_{i}[\vec{x}]\right) Z_{T, t}^{[k]}\left(\vec{x}_{T} \mid \vec{x}\right) \\
& +\sum_{i=1}^{d}\left(\partial_{i}+k \beta_{i}[\vec{x}]\right)\left[D_{i}[\vec{x}]\left(\partial_{i}+k \beta_{i}[\vec{x}]\right) Z_{T, t}^{[k]}\left(\vec{x}_{T} \mid \vec{x}\right)\right] \\
& \equiv \mathcal{F}_{k}^{\dagger} Z_{t, 0}^{[k]}\left(\vec{x} \mid \vec{x}_{0}\right)
\end{aligned}
$$

involving the adjoint operator of Eq. A10

$$
\mathcal{F}_{k}^{\dagger}=k \alpha[\vec{x}]+\sum_{i=1}^{d} F_{i}[\vec{x}]\left(\partial_{i}+k \beta_{i}[\vec{x}]\right)+\sum_{i=1}^{d}\left(\partial_{i}+k \beta_{i}[\vec{x}]\right) D_{i}[\vec{x}]\left(\partial_{i}+k \beta_{i}[\vec{x}]\right)
$$


2. Conditional probability $\mathcal{P}_{t}^{C o n d[k]}(x)$ if starting at $x_{0}$ at time $t=0$ and ending at $x_{T}$ at time $t=T$

Even if it is not a conserved probability, the generating function $Z_{T, 0}^{[k]}\left(x_{T} \mid x_{0}\right)$ satisfies nevertheless some analog of the Chapman-Kolmogorov Eq. 3 as a consequence of the additivity property of Eq. 1

$$
\mathcal{A}[x(0 \leq s \leq T)]=\mathcal{A}[x(0 \leq s \leq t)]+\mathcal{A}[x(t \leq s \leq T)]
$$

that can be plugged into the definition of Eq. A1 to obtain

$$
\begin{aligned}
Z_{T, 0}^{[k]}\left(x_{T} \mid x_{0}\right) & =\left\langle\delta_{x(T), x_{T}} e^{k \mathcal{A}[x(t \leq s \leq T)]}\left[\sum_{x} \delta_{x(t), x}\right] e^{k \mathcal{A}[x(0 \leq s \leq t)]} \delta_{x(0), x_{0}}\right\rangle \\
& =\sum_{x} Z_{T, t}^{[k]}\left(x_{T} \mid x\right) Z_{t, 0}^{[k]}\left(x \mid x_{0}\right)
\end{aligned}
$$

For each $k$, one can thus introduce the conditional probability $\mathcal{P}_{t}^{C o n d[k]}(x)$ to see the value $x$ at the internal time $t \in] 0, T[$

$$
\mathcal{P}_{t}^{C o n d[k]}(x)=\frac{Z_{T, t}^{[k]}\left(x_{T} \mid x\right) Z_{t, 0}^{[k]}\left(x \mid x_{0}\right)}{Z_{T, 0}^{[k]}\left(x_{T} \mid x_{0}\right)}
$$

It is normalized as a consequence of Eq. A15

$$
\sum_{x} \mathcal{P}_{t}^{\text {Cond }[k]}(x)=1
$$

and it satisfies the fixed boundary conditions at time $t=0$ and at time $t=T$

$$
\begin{aligned}
& \mathcal{P}_{0}^{\text {Cond }[k]}(x)=\frac{Z_{T, 0}^{[k]}\left(x_{T} \mid x\right) Z_{0,0}^{[k]}\left(x \mid x_{0}\right)}{Z_{T, 0}^{[k]}\left(x_{T} \mid x_{0}\right)}=\delta_{x, x_{0}} \\
& \mathcal{P}_{T}^{\text {Cond }[k]}(x)=\frac{Z_{T, T}^{[k]}\left(x_{T} \mid x\right) Z_{T, 0}^{[k]}\left(x \mid x_{0}\right)}{Z_{T, 0}^{[k]}\left(x_{T} \mid x_{0}\right)}=\delta_{x, x_{T}}
\end{aligned}
$$

\section{Markov dynamics for the conditional probability $\mathcal{P}_{t}^{C o n d[k]}(x)$}

The Markov dynamics of the conditional probability $\mathcal{P}_{t}^{C o n d[k]}(x)$ can be derived from the Markov dynamics satisfied by the two generating functions in the numerator of Eq. A16, namely :

(i) the forward dynamics of the generating function $Z_{t, 0}^{[k]}\left(x \mid x_{0}\right)$ with respect to its final variable $x$ at time $t$

(ii) the backward dynamics of the generating function $Z_{T, t}^{[k]}\left(x_{T} \mid x\right)$ with respect to its initial variable $x$ at time $t$

a. Forward dynamics of the conditional probability $\mathcal{P}_{t}^{\text {Cond }[k]}(x)$ for discrete-time Markov chains of section III

For the case of discrete-time Markov chains of section III, the conditional probability of Eq. A16 satisfies the forward dynamics

$$
\mathcal{P}_{t+1}^{\text {Cond }[k]}(x)=\sum_{x^{\prime}} W_{t+1 / 2}^{\text {Forw }\left[k ; x_{T}, T\right]}\left(x ; x^{\prime}\right) \mathcal{P}_{t}^{\text {Cond }[k]}\left(x^{\prime}\right)
$$

where the effective probabilities

$$
W_{t+1 / 2}^{F o r w\left[k ; x_{T}, T\right]}\left(x ; x^{\prime}\right)=Z_{T, t+1}^{[k]}\left(x_{T} \mid x\right) W^{[k]}\left(x ; x^{\prime}\right) \frac{1}{Z_{T, t}^{[k]}\left(x_{T} \mid x^{\prime}\right)}
$$

involve the conjugation of the $k$-tilted matrix $W^{[k]}\left(x ; x^{\prime}\right)$ of Eq. A4 with the generating functions $Z_{T, t+1}^{[k]}\left(x_{T} \mid x\right)$ and $Z_{T, t}^{[k]}\left(x_{T} \mid x^{\prime}\right)$ up to the imposed final value $x_{T}$ at time $T$. Eq. A20 is the analog of Eq. 18 concerning the microcanonical conditioning. 
b. Forward dynamics of the conditional probability $\mathcal{P}_{t}^{\text {Cond }[k]}(x)$ for continuous-time Markov jump processes of section IV

For the case of continuous-time Markov jump processes of section IV, the conditional probability of Eq. A16 satisfies the forward dynamics

$$
\partial_{t} \mathcal{P}_{t}^{\text {Cond }[k]}(x)=\sum_{x^{\prime} \neq x}\left[w_{t}^{\text {For } w\left[k ; x_{T}, T\right]}\left(x ; x^{\prime}\right) \mathcal{P}_{t}^{\text {Cond }[k]}\left(x^{\prime}\right)-w_{t}^{\text {Forw }\left[k ; x_{T}, T\right]}\left(x^{\prime} ; x\right) \mathcal{P}_{t}^{C o n d[k]}(x)\right]
$$

where the effective rates

$$
w_{t}^{F o r w\left[k ; x_{T}, T\right]}\left(x ; x^{\prime}\right)=Z_{T, t}^{[k]}\left(x_{T} \mid x\right) w^{k]}\left(x ; x^{\prime}\right) \frac{1}{Z_{T, t}^{[k]}\left(x_{T} \mid x^{\prime}\right)} \text { for } x \neq x^{\prime}
$$

involve the conjugation of the $k$-tilted matrix of Eq. A7 with the generating functions $Z_{T, t+1}^{[k]}\left(x_{T} \mid x\right)$ and $Z_{T, t}^{[k]}\left(x_{T} \mid x^{\prime}\right)$ up to the imposed final value $x_{T}$ at time $T$. Eq. A22 is the analog of Eq. 46 concerning the microcanonical conditioning.

$$
\text { c. } \quad \text { Forward dynamics of the conditional probability } \mathcal{P}_{t}^{\text {Cond }[k]}(\vec{x}) \text { for diffusion processes of section } V
$$

For the case of diffusion processes of section V, the conditional probability of Eq. A16 satisfies the forward dynamics

$$
\partial_{t} \mathcal{P}_{t}^{\text {Cond }[k]}(\vec{x})=-\sum_{i=1}^{d} \partial_{x_{i}}\left[\left(F_{i}[\vec{x}]+F_{i}^{\text {Forw }\left[k ; \vec{x}_{T}, T\right]}[\vec{x} ; t]\right) \mathcal{P}_{t}^{\text {Cond }[k]}(\vec{x})\right]+\sum_{i=1}^{d} \partial_{x_{i}}\left[D_{i}[\vec{x}] \partial_{x_{i}} \mathcal{P}_{t}^{C o n d[k]}(\vec{x})\right]
$$

where the additional time-dependent force

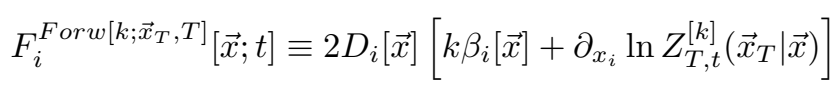

is the analog of Eq. 75 concerning the microcanonical conditioning.

The forward Fokker-Planck dynamics of Eq. A23 can be translated into the following Stratonovich stochastic differential equations for the $d$ components $x_{i}^{*}(t)$ for $i=1, \ldots, d$ in terms of $d$ independent Brownian motions $B_{i}(t)$

$$
d x_{i}^{*}(t)=\left(f_{i}\left[\vec{x}^{*}(t)\right]+F_{i}^{F o r w\left[k ; \vec{x}_{T}, T\right]}[\vec{x} ; t]\right) d t+g_{i}\left[\vec{x}^{*}(t)\right] d B_{i}(t)
$$

where the only differences with respect to the unconditioned case of Eq. 61 are the additional time-dependent forces $F_{i}^{F o r w ~}\left[k ; \vec{x}_{T}, T\right][\vec{x} ; t]$ given in Eq. A24.

\section{Appendix B: Reminder on the conditioning for large $T$ when there is a normalizable steady state $P_{s t}(x)$}

In this Appendix, the Markov processes $x(t)$ is assumed to converge towards some normalizable steady-state $P_{s t}(x)$. This steady state $P_{s t}(x)$ can be interpreted as the positive eigenvector $\left\langle x \mid r_{0}\right\rangle=r_{0}(x)$ associated to the highest eigenvalue of the Markov generator

$$
P_{s t}(x)=\left\langle x \mid r_{0}\right\rangle=r_{0}(x)
$$

while the corresponding positive left eigenvector is constant

$$
\left\langle l_{0} \mid x\right\rangle=l_{0}(x)=1
$$

When the time interval $\left(t-t_{0}\right)$ becomes large, the propagator $P_{t, t_{0}}\left(x, x_{0}\right)$ is dominated by this highest eigenvalue contribution

$$
P_{t, t_{0}}\left(x, x_{0}\right) \underset{\left(t-t_{0}\right) \rightarrow+\infty}{\simeq}\left\langle x \mid r_{0}\right\rangle\left\langle l_{0} \mid x_{0}\right\rangle=r_{0}(x) l_{0}(x)=P_{s t}(x)
$$

and describes the convergence towards the steady state $P_{s t}(x)$ for any initial condition $x_{0}$. 
1. Asymptotic analysis of the generating function $Z_{t, t_{0}}^{[k]}\left(x \mid x_{0}\right)$ for large time interval $\left(t-t_{0}\right)$

For $k=0$, the generating function of Eq. A1 coincides with the propagaor $P_{t, t_{0}}\left(x, x_{0}\right)$ discussed above

$$
Z_{t, t_{0}}^{[k=0]}\left(x \mid x_{0}\right)=P_{t, t_{0}}\left(x, x_{0}\right)
$$

As a consequence for $k \neq 0$, at least in some region around $k=0$, one expects that for large time-interval $\left(t-t_{0}\right)$, the generating function will be similarly dominated by the contribution of the highest eigenvalue of the appropriate $k$-deformed generator

$$
Z_{t, t_{0}}^{[k]}\left(x \mid x_{0}\right) \underset{\left(t-t_{0}\right) \rightarrow+\infty}{\simeq} e^{\left(t-t_{0}\right) G(k)}\left\langle x \mid r_{k}\right\rangle\left\langle l_{k} \mid x_{0}\right\rangle=e^{\left(t-t_{0}\right) G(k)} r_{k}(x) l_{k}\left(x_{0}\right)
$$

with its positive right eigenvector $r_{k}(x) \geq 0$ and its positive left eigenvector $l_{k}(x) \geq 0$ satisfying the normalization

$$
1=\left\langle l_{k} \mid r_{k}\right\rangle=\sum_{x}\left\langle l_{k} \mid x\right\rangle\left\langle x \mid r_{k}\right\rangle=\sum_{x} r_{k}(x) l_{k}(x)
$$

while $\left[\left(t-t_{0}\right) G(k)\right]$ represents the generating function of the cumulants of the time-additive observable $A_{t, t_{0}}$, i.e. $G(k)$ corresponds to the scaled cumulants generating function in the large deviations theory, as recalled in more details below in subsection B 3 .

\section{Asymptotic analysis of the conditional probability $\mathcal{P}_{t}^{C o n d[k]}(x)$ at some interior time $0 \ll t \ll T$}

For large $T$, if one is interested at some interior time $t$ satisfying $0 \ll t \ll T$, one can plug the asymptotic behavior of Eq. B5 into the three generating functions of Eq. A16 to obtain the asymptotic behavior of the conditional probability

$$
\mathcal{P}_{t}^{C o n d[k]}(x) \underset{0 \ll t \ll T}{\simeq} \frac{e^{(T-t) G(k)} r_{k}\left(x_{T}\right) l_{k}(x) e^{t G(k)} r_{k}(x) l_{k}\left(x_{0}\right)}{e^{T G(k)} r_{k}\left(x_{T}\right) l_{k}\left(x_{0}\right)}=l_{k}(x) r_{k}(x)
$$

Since it is independent of the interior time $t$ as long as $0 \ll t \ll T$, it is useful to introduce the notation

$$
\rho_{k}(x) \equiv l_{k}(x) r_{k}(x)
$$

for the stationary density of the conditional probability $\mathcal{P}_{t}^{C o n d[k]}(x)$ in the interior time region $0 \ll t \ll T$.

\section{Physical meaning of the canonical $k$-conditioning in terms of the large deviations properties of $A(t)$}

Since the time-additive observable $A(t)$ of Eq. 1 is extensive with respect to the time-interval, it is useful to introduce its rescaled intensive counterpart

$$
a_{t, t_{0}} \equiv \frac{A(t)-A\left(t_{0}\right)}{t-t_{0}}=\frac{A\left[x\left(t_{0} \leq s \leq t\right)\right]}{t-t_{0}}
$$

that will converge towards its steady value $a_{s t}$ that can be computed from the steady state $P_{s t}(x)$ and from the corresponding steady flows

$$
a_{t, t_{0}} \underset{\left(t-t_{0}\right) \rightarrow \infty}{\simeq} a_{s t}
$$

The probability $P_{t, t_{0}}(a)$ to see the value $a$ different from this steady value $a_{s t}$ displays the large deviations form with respect to the time interval $\left(t-t_{0}\right)$

$$
P_{t, t_{0}}(a) \underset{\left(t-t_{0}\right) \rightarrow+\infty}{\simeq} e^{-\left(t-t_{0}\right) I(a)}
$$

where the positive rate function $I(a) \geq 0$ vanishes only for the steady value $a_{s t}$ of Eq. B10

$$
I\left(a_{s t}\right)=0
$$


The generating function of the additive observable $A\left[x\left(t_{0} \leq s \leq t\right)=A(t)-A\left(t_{0}\right)=\left(t-t_{0}\right) a_{t, t_{0}}\right.$ can be evaluated from Eq. B11 via the saddle-point method for large $\left(t-t_{0}\right)$

$$
\left\langle e^{k A\left[x\left(t_{0} \leq s \leq t\right)\right.}\right\rangle=\left\langle e^{k\left(t-t_{0}\right) a_{t, t_{0}}}\right\rangle \equiv \int d a e^{k\left(t-t_{0}\right) a} P_{t, t_{0}}(a) \underset{\left(t-t_{0}\right) \rightarrow+\infty}{\simeq} \int d a e^{\left(t-t_{0}\right)[k a-I(a)]} \underset{\left(t-t_{0}\right) \rightarrow+\infty}{\simeq} e^{\left(t-t_{0}\right) G(k)}
$$

So the scaled cumulants generating function $G(k)$ that has been introduced in Eq. B5 is the Legendre transform of the rate function $I(a)$

$$
\begin{aligned}
k a-I(a) & =G(k) \\
k-I^{\prime}(a) & =0
\end{aligned}
$$

while the reciprocal Legendre transform reads

$$
\begin{aligned}
k a-G(k) & =I(a) \\
a-G^{\prime}(k) & =0
\end{aligned}
$$

As a consequence, the canonical $k$-conditioning discussed around Eq. B8 can be considered as asymptotically equivalent to the microcanonical conditioning on the intensive additive variable at the corresponding Legendre value $a=G^{\prime}(k)$ of Eq. B15.

\section{Corresponding time-independent generators of the conditioned dynamics for $1 \ll t \ll T$}

a. Forward dynamics of the conditional probability $\mathcal{P}_{t}^{\text {Cond }[k]}(x)$ for discrete-time Markov chains of section III

For the case of discrete-time Markov chains of section III, the asymptotic form of Eq. B5 for the generating function yields that the effective probabilities of Eq. A20 become time-independent in the regime $1 \ll t \ll T$

$$
\begin{aligned}
W_{t+1 / 2}^{F o r w\left[k ; x_{T}, T\right]}\left(x ; x^{\prime}\right) & \underset{1 \ll i t}{\simeq} e^{(T-t-1) G(k)} r_{k}\left(x_{T}\right) l_{k}(x) W^{[k]}\left(x ; x^{\prime}\right) \frac{1}{e^{(T-t) G(k)} r_{k}\left(x_{T}\right) l_{k}\left(x^{\prime}\right)} \\
& \underset{1 \ll i \ll T}{\simeq} e^{-G(k)} l_{k}(x) W^{[k]}\left(x ; x^{\prime}\right) \frac{1}{l_{k}\left(x^{\prime}\right)}
\end{aligned}
$$

where $e^{G(k)}$ is the highest eigenvalue of the $k$-tilted matrix $W^{[k]}\left(x ; x^{\prime}\right)$ of Eq. A4, while $l_{k}($.$) is the corresponding$ positive eigenvector

$$
e^{G(k)} l_{k}\left(x^{\prime}\right)=\sum_{x} l_{k}(x) W^{[k]}\left(x ; x^{\prime}\right)
$$

The corresponding positive right eigenvector $r_{k}($.

$$
e^{G(k)} r_{k}(x)=\sum_{x^{\prime}} W^{[k]}\left(x ; x^{\prime}\right) r_{k}\left(x^{\prime}\right)
$$

appears in the conditioned steady state of Eq. B8 together with the left eigenvector $l_{k}($.$) .$

b. Forward dynamics of the conditional probability $\mathcal{P}_{t}^{\text {Cond }[k]}(x)$ for continuous-time Markov jump processes of section IV

For the case of continuous-time Markov jump processes of section IV, the asymptotic form of Eq. B5 for the generating function yields that the effective rates of Eq. A22 become time-independent in the regime $1 \ll t \ll T$

$$
w_{t}^{F o r w\left[k ; x_{T}, T\right]}\left(x ; x^{\prime}\right) \underset{1 \ll t \ll T}{\simeq} l_{k}(x) w^{k]}\left(x ; x^{\prime}\right) \frac{1}{l_{k}\left(x^{\prime}\right)} \text { for } x \neq x^{\prime}
$$

where $l_{k}($.$) is the positive eigenvector associated to the highest eigenvalue G(k)$ of the $k$-tilted matrix $w^{[k]}\left(x ; x^{\prime}\right)$ of Eq. A7

$$
G(k) l_{k}\left(x^{\prime}\right)=\sum_{x} l_{k}(x) w^{[k]}\left(x ; x^{\prime}\right)=l_{k}\left(x^{\prime}\right) w^{[k]}\left(x^{\prime} ; x^{\prime}\right)+\sum_{x \neq x^{\prime}} l_{k}(x) w^{[k]}\left(x ; x^{\prime}\right)
$$


Via the conservation of probability, the diagonal element can be computed in terms of the off-diagonal elements of Eq. B19 using the eigenvalue Eq. B20

$$
\begin{aligned}
& w_{t}^{F \text { orw }\left[k ; x_{T}, T\right]}\left(x^{\prime} ; x^{\prime}\right)=-\sum_{x \neq x^{\prime}} w_{t}^{F o r w\left[k ; x_{T}, T\right]}\left(x ; x^{\prime}\right) \underset{1 \ll t \ll T}{\simeq}-\left[\sum_{x \neq x^{\prime}} l_{k}(x) w^{k]}\left(x ; x^{\prime}\right)\right] \frac{1}{l_{k}\left(x^{\prime}\right)} \\
& =-\left[G(k) l_{k}\left(x^{\prime}\right)-l_{k}\left(x^{\prime}\right) w^{[k]}\left(x^{\prime} ; x^{\prime}\right)\right] \frac{1}{l_{k}\left(x^{\prime}\right)}=w^{[k]}\left(x^{\prime} ; x^{\prime}\right)-G(k)
\end{aligned}
$$

so that it involves the diagonal element $w^{[k]}\left(x^{\prime} ; x^{\prime}\right)$ and the eigenvalue $G(k)$.

The positive right eigenvector $r_{k}($.$) of the k$-tilted matrix $w^{[k]}\left(x ; x^{\prime}\right)$

$$
G(k) r_{k}(x)=\sum_{x^{\prime}} w^{[k]}\left(x ; x^{\prime}\right) r_{k}\left(x^{\prime}\right)
$$

appears in the conditioned steady state of Eq. B8 together with the left eigenvector $l_{k}($.$) .$

c. $\quad$ Forward dynamics of the conditional probability $\mathcal{P}_{t}^{\text {Cond }[k]}(\vec{x})$ for diffusion processes of section $V$

For the case of diffusion processes of section V, the asymptotic form of Eq. B5 for the generating function yields that the effective additional force of Eq. A24 becomes time-independent in the regime $1 \ll t \ll T$

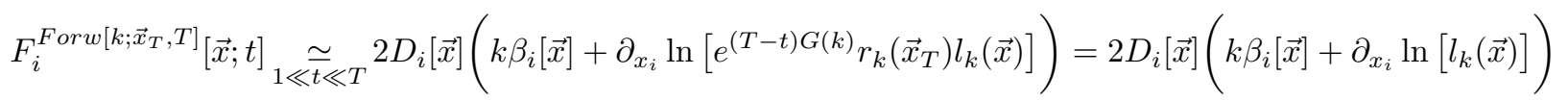

where $l_{k}($.$) is the positive eigenvector associated to the highest eigenvalue G(k)$ of the adjoint differential operator $\mathcal{F}_{k}^{\dagger}$ of Eq. A13

$$
\begin{aligned}
G(k) l_{k}(\vec{x}) & =\mathcal{F}_{k}^{\dagger} l_{k}(\vec{x}) \\
& =k \alpha[\vec{x}] l_{k}(\vec{x})+\sum_{i=1}^{d} F_{i}[\vec{x}]\left(\partial_{i}+k \beta_{i}[\vec{x}]\right) l_{k}(\vec{x})+\sum_{i=1}^{d}\left(\partial_{i}+k \beta_{i}[\vec{x}]\right)\left[D_{i}[\vec{x}]\left(\partial_{i}+k \beta_{i}[\vec{x}]\right) l_{k}(\vec{x})\right]
\end{aligned}
$$

The corresponding positive eigenvector $r_{k}($.$) of the operator \mathcal{F}_{k}$ of Eq. A13

$$
\begin{aligned}
G(k) r_{k}(\vec{x}) & =\mathcal{F}_{k} r_{k}(\vec{x}) \\
& =k \alpha[\vec{x}] r_{k}(\vec{x})-\sum_{i=1}^{d}\left(\partial_{i}-k \beta_{i}[\vec{x}]\right)\left[F_{i}[\vec{x}] r_{k}(\vec{x})\right]+\sum_{i=1}^{d}\left(\partial_{i}-k \beta_{i}[\vec{x}]\right)\left[D_{i}[\vec{x}]\left(\partial_{i}-k \beta_{i}[\vec{x}]\right) r_{k}(\vec{x})\right]
\end{aligned}
$$

appears in the conditioned steady state of Eq. B8 together with the left eigenvector $l_{k}($.$) .$

[1] Y. Oono, Progress of Theoretical Physics Supplement 99, 165 (1989).

[2] R.S. Ellis, Physica D 133, 106 (1999).

[3] H. Touchette, Phys. Rep. 478, 1 (2009).

[4] A. de La Fortelle, PhD (2000) "Contributions to the theory of large deviations and applications" INRIA Rocquencourt.

[5] G. Fayolle and A. de La Fortelle, Problems of Information Transmission 38, 354 (2002).

[6] C. Monthus, Eur. Phys. J. B 92, 149 (2019) in the topical issue " Recent Advances in the Theory of Disordered Systems" edited by F. Igloi and H. Rieger.

[7] C. Monthus, J. Stat. Mech. (2021) 033201.

[8] C. Monthus, J. Stat. Mech. (2021) 063211.

[9] A. de La Fortelle, Problems of Information Transmission 37,120 (2001).

[10] C. Maes and K. Netocny, Europhys. Lett. 82, 30003 (2008).

[11] C. Maes, K. Netocny and B. Wynants, Markov Proc. Rel. Fields. 14, 445 (2008).

[12] B. Wynants, arXiv:1011.4210, PhD Thesis (2010), "Structures of Nonequilibrium Fluctuations", Catholic University of Leuven. 
[13] A. C. Barato and R. Chétrite, J. Stat. Phys. 160, 1154 (2015).

[14] L. Bertini, A. Faggionato and D. Gabrielli, Ann. Inst. Henri Poincare Prob. and Stat. 51, 867 (2015).

[15] L. Bertini, A. Faggionato and D. Gabrielli, Stoch. Process. Appli. 125, 2786 (2015).

[16] R. Chétrite, HDR Thesis (2018) "Pérégrinations sur les phénomènes aléatoires dans la nature", Laboratoire J.A. Dieudonné, Université de Nice.

[17] C. Monthus, J. Stat. Mech. (2019) 023206.

[18] C. Monthus, J. Phys. A: Math. Theor. 52, 135003 (2019).

[19] C. Monthus, J. Phys. A: Math. Theor. 52, 025001 (2019).

[20] C. Monthus, J. Phys. A: Math. Theor. 52, 485001 (2019).

[21] A. C. Barato, R. Chétrite, J. Stat. Mech. (2018) 053207.

[22] L. Chabane, R. Chétrite, G. Verley, J. Stat. Mech. (2020) 033208.

[23] C. Monthus, J. Stat. Mech. (2021) 083212.

[24] C. Monthus, J. Stat. Mech. (2021) 083205.

[25] C. Monthus, J. Stat. Mech. (2021) 103202.

[26] C. Monthus, J. Stat. Mech. (2022) 013206.

[27] C. Monthus, arXiv:2109.05924.

[28] C. Monthus, J. Stat. Mech. (2021) 123205.

[29] C. Maes, K. Netocny and B. Wynants Physica A 387, 2675 (2008).

[30] J. Hoppenau, D. Nickelsen and A. Engel, New J. Phys. 18083010 (2016).

[31] C. Monthus, J. Stat. Mech. (2021) 033303.

[32] C. Giardina, J. Kurchan and L. Peliti, Phys. Rev. Lett. 96, 120603 (2006).

[33] B. Derrida, J. Stat. Mech. P07023 (2007).

[34] C. Giardina, J. Kurchan, V. Lecomte and J. Tailleur, J. Stat. Phys. 145, 787 (2011).

[35] R. L. Jack, P. Sollich, The European Physical Journal Special Topics 224, 2351 (2015).

[36] A. Lazarescu, J. Phys. A: Math. Theor. 48503001 (2015).

[37] A. Lazarescu, J. Phys. A: Math. Theor. 50254004 (2017).

[38] R. L. Jack, Eur. Phy. J. B 93, 74 (2020).

[39] V. Lecomte, PhD Thesis (2007) "Thermodynamique des histoires et fluctuations hors d'équilibre" Université Paris.

[40] V. Lecomte, C. Appert-Rolland and F. van Wijland, Phys. Rev. Lett. 95, 010601 (2005).

[41] V. Lecomte, C. Appert-Rolland and F. van Wijland, J. Stat. Phys. 127, 51 (2007).

[42] V. Lecomte, C. Appert-Rolland and F. van Wijland, Comptes Rendus Physique 8, 609 (2007).

[43] J.P. Garrahan, R.L. Jack, V. Lecomte, E. Pitard, K. van Duijvendijk, F. van Wijland, Phys. Rev. Lett. 98,195702 (2007).

[44] J.P. Garrahan, R.L. Jack, V. Lecomte, E. Pitard, K. van Duijvendijk and F. van Wijland, J. Phys. A 42, 075007 (2009).

[45] K. van Duijvendijk, R.L. Jack and F. van Wijland, Phys. Rev. E 81, 011110 (2010).

[46] R. L. Jack, P. Sollich, Prog. Theor. Phys. Supp. 184, 304 (2010).

[47] D. Simon, J. Stat. Mech. (2009) P07017.

[48] V. Popkov, G. M. Schuetz, D. Simon, J. Stat. Mech. P10007 (2010).

[49] D. Simon, J. Stat. Phys. 142, 931 (2011).

[50] V. Popkov, G. M. Schuetz, J. Stat. Phys 142, 627 (2011)

[51] V. Belitsky, G. M. Schuetz, J. Stat. Phys. 152, 93 (2013).

[52] O. Hirschberg, D. Mukamel, G. M. Schuetz, J. Stat. Mech. P11023 (2015).

[53] G. M. Schuetz, From Particle Systems to Partial Differential Equations II, Springer Proceedings in Mathematics and Statistics Volume 129, pp 371-393, P. Goncalves and A.J. Soares (Eds.), (Springer, Cham, 2015).

[54] R. Chétrite and H. Touchette, Phys. Rev. Lett. 111, 120601 (2013).

[55] R. Chétrite and H. Touchette Ann. Henri Poincare 16, 2005 (2015).

[56] R. Chétrite, H. Touchette, J. Stat. Mech. P12001 (2015).

[57] P. T. Nyawo, H. Touchette, Phys. Rev. E 94, 032101 (2016).

[58] H. Touchette, Physica A 504, 5 (2018).

[59] F. Angeletti, H. Touchette, Journal of Mathematical Physics 57, 023303 (2016).

[60] P. T. Nyawo, H. Touchette, Europhys. Lett. 116, 50009 (2016);

P. T. Nyawo, H. Touchette, Phys. Rev. E 98, 052103 (2018).

[61] J. P. Garrahan, Physica A 504, 130 (2018).

[62] E. Roldan and P. Vivo, Phys. Rev. E 100, 042108 (2019).

[63] A. Lazarescu, T. Cossetto, G. Falasco and M. Esposito, J. Chem. Phys. 151, 064117 (2019).

[64] B. Derrida and T. Sadhu, Journal of Statistical Physics 176, 773 (2019);

B. Derrida and T. Sadhu, Journal of Statistical Physics 177, 151 (2019).

[65] K. Proesmans, B. Derrida, J. Stat. Mech. (2019) 023201.

[66] N. Tizon-Escamilla, V. Lecomte and E. Bertin, J. Stat. Mech. (2019) 013201.

[67] J. du Buisson, H. Touchette, Phys. Rev. E 102, 012148 (2020).

[68] E. Mallmin, J. du Buisson and H. Touchette, J. Phys. A: Math. Theor. 54295001 (2021).

[69] F. Carollo, J. P. Garrahan, I. Lesanovsky, C. Perez-Espigares, Phys. Rev. A 98, 010103 (2018).

[70] F. Carollo, R. L. Jack, J. P. Garrahan, Phys. Rev. Lett. 122, 130605 (2019).

[71] F. Carollo, J. P. Garrahan, R. L. Jack, J. Stat. Phys. 184, 13 (2021).

[72] C. Monthus, J. Stat. Mech. (2021) 063301. 
[73] A. Lapolla, D. Hartich, A. Godec, Phys. Rev. Research 2, 043084 (2020).

[74] L. Chabane, A. Lazarescu and G. Verley, arXiv:2109.06830

[75] A. Mazzolo, J. Stat. Mech. (2017) 023203.

[76] A. Mazzolo, J. Math. Phys. 58, 0953302 (2017).

[77] P. Garbaczewski, Phys. Rev. E 96, 032104 (2017).

[78] A. Mazzolo, J. Stat. Mech. (2018) 073204.

[79] C. Larmier, A. Mazzolo and A. Zoia, J. Stat. Mech. (2019) 113208.

[80] B. De Bruyne, S. N. Majumdar, H. Orland and G. Schehr, J. Stat. Mech. (2021) 123204.

[81] H. Orland, J. Chem. Phys. 134, 174114 (2011).

[82] S.N. Majumdar and H. Orland, J. Stat. Mech. 6, 06039 (2015).

[83] M. Delarue, P. Koehl and H. Orland, J. Chem. Phys. 147, 152703 (2017).

[84] B. De Bruyne, S.N. Majumdar and G. Schehr, Phys. Rev. E 104, 024117 (2021).

[85] B. De Bruyne, S.N. Majumdar and G. Schehr, J. Phys. A: Math. Theor. 54385004 (2021).

[86] J. Grela, S. N. Majumdar and G. Schehr, J. Stat. Phys. 183, 1 (2021).

[87] M. R. Evans, S. N. Majumdar, G. Schehr, J. Phys. A: Math. Theor. 53193001 (2020).

[88] M. Montero and J. Villarroel, PRE 94, 032132 (2016). 